TRANSACTIONS OF THE

AMERICAN MATHEMATICAL SOCIETY

Volume 353, Number 8, Pages 3115-3147

S 0002-9947(01)02723-4

Article electronically published on April 10, 2001

\title{
COMPUTING THE HOMOLOGY OF KOSZUL COMPLEXES
}

\author{
BERNHARD KÖCK
}

\begin{abstract}
Let $R$ be a commutative ring and $I$ an ideal in $R$ which is locally generated by a regular sequence of length $d$. Then, each f. g. projective $R / I$ module $V$ has an $R$-projective resolution $P$. of length $d$. In this paper, we compute the homology of the $n$-th Koszul complex associated with the homomorphism $P_{1} \rightarrow P_{0}$ for all $n \geq 1$, if $d=1$. This computation yields a new proof of the classical Adams-Riemann-Roch formula for regular closed immersions which does not use the deformation to the normal cone any longer. Furthermore, if $d=2$, we compute the homology of the complex $N \operatorname{Sym}^{2} \Gamma(P$.) where $\Gamma$ and $N$ denote the functors occurring in the Dold-Kan correspondence.
\end{abstract}

\section{INTRODUCTION}

In the theory of exterior powers of $\mathrm{f}$. g. projective modules over a commutative ring $R$, there are various formulas which, so far, can only be proved by using geometric means (e.g., the projective fibre bundle or blowing up). An example is the formula which computes the composition of exterior powers in the Grothendieck ring $K_{0}(R)$ (e.g., see [FL]). The plethysm problem is to find (explicit) functorial short exact sequences which prove this formula within the framework of commutative algebra. It has been intensively studied in universal representation theory. In this paper, we study the analogous problem for the Adams-Riemann-Roch formula for regular closed immersions (between affine schemes).

We now recall the Adams-Riemann-Roch formula (see [FL]). Let $i: Y \hookrightarrow X$ be a regular closed immersion of schemes with the locally free conormal sheaf $\mathcal{C}$. Let $\psi_{n}: K_{0}(X) \rightarrow K_{0}(X)$ denote the $n$-th Adams operation on the Grothendieck ring $K_{0}(X)$; it is defined as a certain integral polynomial in the exterior power operations on $K_{0}(X)$. For any locally free $\mathcal{O}_{Y}$-module $\mathcal{V}$, we choose a locally free resolution $\mathcal{P}$. of $i_{*}(\mathcal{V})$ on $X$, and we map the element $[\mathcal{V}] \in K_{0}(Y)$ to the Euler characteristic $\sum_{k \geq 0}(-1)^{k}\left[\mathcal{P}_{k}\right] \in K_{0}(X)$. This association induces a well-defined homomorphism $i_{*}: K_{0}(Y) \rightarrow K_{0}(X)$. The Adams-Riemann-Roch theorem for $i_{*}$ now states that, for all $y \in K_{0}(Y)$, we have

$$
\psi_{n}\left(i_{*}(y)\right)=i_{*}\left(\theta^{n}(\mathcal{C}) \cdot \psi_{n}(y)\right) \text { in } K_{0}(X) ;
$$

here, $\theta^{n}(\mathcal{C})$ denotes the so-called Bott element associated with $\mathcal{C}$ (see $[\overline{\mathrm{FL}})$.

This formula (for only one $n \geq 2$ ) implies the famous Grothendieck-RiemannRoch theorem for $i_{*}$ in a rather formal manner (see [FL]). It has been formulated

Received by the editors May 30, 1999 and, in revised form, January 30, 2000.

2000 Mathematics Subject Classification. Primary 13D25, 19E20, 14C40, 13D15.

Key words and phrases. Koszul complex, Dold-Kan correspondence, cross effect functor, symmetric power operation, Adams-Riemann-Roch theorem, plethysm problem. 
for the first time by Manin in $[\mathbf{M}]$, but it has been proved there only up to $n^{2}$ torsion in $K_{0}(X)$. Moreover, as a formula in $K_{0}(X) \otimes \mathbb{Q}$, it follows already from Théorème 4.3 in Expose VII of [SGA 6]. The given version without denominators can be derived from Théorème 2.1 in Jouanolou's paper [J] and can be found in Soulé's paper [S] and in the book [FL] by Fulton and Lang. In all these sources, the deformation to the normal cone is the crucial tool in the proof. The object of this paper is to construct explicit short exact sequences of $\mathcal{O}_{X}$-modules which prove this formula in the case $\operatorname{codim}(Y / X)=1, n$ arbitrary and in the case $\operatorname{codim}(Y / X)=2$, $n=2$. Since the proof of the Adams-Riemann-Roch formula in the general case can immediately be reduced to the case $\operatorname{codim}(Y / X)=1$ using blowing up and the excess intersection formula (see [SGA 6]), we in particular obtain a new, very natural and simple proof in the general case which, in contrast to [J], [S], or [FL], does not use the deformation any longer.

To illustrate our approach, we now describe it rather completely for $\operatorname{codim}(Y / X)$ $=1$ and $n=2$. As in the beginning of the introduction, we consider only the affine case; so, let $X=\operatorname{Spec}(R)$ and $Y=\operatorname{Spec}(R / I)$ where the ideal $I$ of $R$ is locally generated by a nonzero-divisor. Let $\sigma_{2}$ and $\lambda_{2}$ denote the second symmetric power and second exterior power operation. Let $V$ be an $\mathrm{f}$. g. projective $R / I$-module and

$$
0 \rightarrow P \rightarrow Q \rightarrow V \rightarrow 0
$$

an $R$-projective resolution of $V$. Since $\psi_{2}=\sigma_{2}-\lambda_{2}$ and $\theta^{2}\left(I / I^{2}\right)=1+\left[I / I^{2}\right]$, it suffices to prove the formula

$$
\sigma_{2}\left(i_{*}([V])\right)=i_{*}\left(\left[\operatorname{Sym}^{2}(V)\right]-\left[\Lambda^{2}(V) \otimes I / I^{2}\right]\right) \quad \text { in } \quad K_{0}(X)
$$

and a similar formula for $\lambda_{2}$. Now, the main idea is to consider the Koszul complex

$$
K: \quad 0 \rightarrow \Lambda^{2}(P) \rightarrow P \otimes Q \rightarrow \operatorname{Sym}^{2}(Q) \rightarrow 0
$$

which, for instance, can be defined as a subcomplex of the natural short exact sequence $0 \rightarrow \Lambda^{2}(Q) \rightarrow Q \otimes Q \rightarrow \operatorname{Sym}^{2}(Q) \rightarrow 0$. It is immediately clear that the alternating sum of the objects of this complex equals the left-hand side of the formula (0.1). Since the alternating sum of the objects equals the alternating sum of the homology modules, it suffices to show that $H_{0}(K) \cong \operatorname{Sym}^{2}(V)$, $H_{1}(K) \cong \Lambda^{2}(V) \otimes I / I^{2}$, and $H_{2}(K) \cong 0$. (Here, $H_{0}(K), H_{1}(K)$, and $H_{2}(K)$ denote the homology of $K$ at the places $\operatorname{Sym}^{2}(Q), P \otimes Q$, and $\Lambda^{2}(P)$, respectively.) Whilst the isomorphisms $H_{0}(K) \cong \operatorname{Sym}^{2}(V)$ and $H_{2}(K) \cong 0$ can be proved rather easily (see Remark 3.3), the proof of the isomorphism $H_{1}(K) \cong \Lambda^{2}(V) \otimes I / I^{2}$ is more complicated. The most important observation for this is the fact that $H_{1}(K)$ does not depend on the chosen resolution and that the association $V \mapsto H_{1}(K)=: F(V)$ is even a functor. This can be proved as follows. Any homomorphism $V \rightarrow V^{\prime}$ between $\mathrm{f}$. g. projective $R / I$-modules can be lifted to a homomorphism from a resolution of $V$ to a resolution of $V^{\prime}$, and this lifted homomorphism then induces a homomorphism between the corresponding Koszul complexes $K$ and $K^{\prime}$ in the obvious way. In order to prove that the induced homomorphism between the homology modules does not depend on the lifting, we consider the complex

$$
L: \quad 0 \rightarrow P \otimes P \rightarrow \operatorname{Sym}^{2}(P) \oplus P \otimes Q \rightarrow \operatorname{Sym}^{2}(Q) \rightarrow 0
$$

(with the obvious differentials) which is quasi-isomorphic to the complex $K$. One can show (see also Remark 2.6) that any homotopy between two homomorphisms 
between the resolutions induces a homotopy between the corresponding homomorphisms between the complexes $L$ and $L^{\prime}$. (The analogous statement for the complexes $K$ and $K^{\prime}$ is not true!) As usual in homological algebra, this implies that the association $V \mapsto H_{1}(L) \cong F(V)$ is a well-defined functor. In particular, for any f. g. projective $R / I$-modules $V, W$, the direct sum $F(V) \oplus F(W)$ is a canonical direct summand of $F(V \oplus W)$. Let $\mathrm{cr}_{2}(F)(V, W)$ denote the complement. Then $\mathrm{cr}_{2}(F)$ is a bifunctor. It is called the second cross effect of $F$. (This terminology has been introduced by Eilenberg and Mac Lane in [EM].) One easily sees that we have $\mathrm{cr}_{2}(F) \cong \operatorname{Tor}_{1}^{R}$ in our situation. Hence, $\operatorname{cr}_{2}(F)$ is biadditive, and we have $\operatorname{cr}_{2}(F)(R / I, R / I) \cong I / I^{2}$. Furthermore, one immediately sees that $F(R / I) \cong 0$. Eilenberg $([\overline{\mathrm{E}})$ and Watts $(\overline{\mathrm{Wa}}])$ have proved that any additive functor $F$ on the category of $\mathrm{f}$. g. projective modules over a commutative ring $A$ is isomorphic to the tensor functor $F(A) \otimes-$. Similarly, one easily shows that any functor $F$ with the three properties just proved is isomorphic to the functor $\Lambda^{2}(-) \otimes I / I^{2}$ (see Corollary 1.6). This finally proves the claimed isomorphism $H_{1}(K) \cong \Lambda^{2}(V) \otimes I / I^{2}$ and hence the formula (0.1). The analogous formula for $\lambda_{2}$ can be proved in a similar manner (see also Remark 3.6).

Now we briefly describe the idea of our approach in the case $\operatorname{codim}(Y / X)=2$, $n=2$. Let $\mathcal{V}$ be a locally free $\mathcal{O}_{Y}$-module and $\mathcal{P}$. a locally free resolution of $i_{*}(\mathcal{V})$ on $X$. Here, we compute the homology of the complex $\mathcal{L}:=N \operatorname{Sym}^{2} \Gamma(\mathcal{P}$.) where $\Gamma$ and $N$ denote the functors occurring in the Dold-Kan correspondence (see section 2). The idea to consider this complex is suggested by the observation that the complex $L$ used in the case $\operatorname{codim}(Y / X)=1$ is isomorphic to the complex $N \operatorname{Sym}^{2} \Gamma(P \rightarrow Q)$ (see section 2). On the contrary, it seems that an analogue of the Koszul complex $K$ does not exist in the case $\operatorname{codim}(Y / X) \geq 2$. We obtain the following result (see Theorem 6.4): $H_{0}(\mathcal{L}) \cong i_{*}\left(\operatorname{Sym}^{2}(\mathcal{V})\right), H_{1}(\mathcal{L}) \cong i_{*}\left(\Lambda^{2}(\mathcal{V}) \otimes \mathcal{C}\right), H_{2}(\mathcal{L}) \cong i_{*}\left(D^{2}(\mathcal{V}) \otimes\right.$ $\left.\Lambda^{2}(\mathcal{C})\right)$, and $H_{k}(\mathcal{L}) \cong 0$ for $k \geq 3$. This computation of the homology then implies the Adams-Riemann-Roch formula in the case $\operatorname{codim}(Y / X)=2, n=2$ as above (see section 6). The main tools we use in this computation are the EilenbergZilber theorem and the universal form of the Cauchy decomposition developed by Akin, Buchsbaum and Weyman in [ABW]. We again verify certain properties of the corresponding cross effect functors which, together with easy abstract lemmas, imply the above isomorphisms.

In section 5, we present a further application of the theory of cross effect functors developed in section 1. We prove the Riemann-Roch formula for tensor power operations (see Theorem 5.3) which has been proved in the paper [K2] using the deformation to the normal cone (and which in turn implies the Adams-RiemannRoch formula in characteristic 0 ; see section 4 in $[\mathrm{K} 2]$ ). Here, the codimension of $Y$ in $X$ may be arbitrary. For any locally free $\mathcal{O}_{Y}$-module $\mathcal{V}$ and for any locally free resolution $\mathcal{P}$. $\rightarrow i_{*}(\mathcal{V})$ of $i_{*}(\mathcal{V})$ on $X$, we compute the homology of the total complex of the $n$-th tensor power $\mathcal{P} .{ }^{\otimes n}$ of $\mathcal{P}$. together with the canonical action of the symmetric group (see Theorem 5.1).

Acknowledgments. I would like to thank D. Grayson and R. McCarthy for many very helpful discussions where I have learned, for example, the (advantages of) simplicial techniques. In particular, D. Grayson has contributed the basic idea for the important Corollary 2.5. Furthermore, R. McCarthy has explained the main features of cross effect functors to me. It is a pleasure to thank them for all this assistance. 


\section{ON CROSS EFFECT FUNCTORS}

First, we recall the definition of the cross effect functors $\mathrm{cr}_{k}(F), k \geq 0$, associated with a functor $F$ between additive categories (cf. [EM] and [JM]). Then, we introduce the diagonal and plus maps and describe these maps explicitly for the second and third symmetric power functor. The most important result of this section is Theorem 1.5 which states that any functor $F$ (of finite degree) from the category of f. g. projective modules over a ring $A$ to any abelian category is essentially determined by the objects $\operatorname{cr}_{k}(F)(A, \ldots, A), k \geq 0$, and the diagonal and plus maps between these objects. Finally, we characterize the classical exterior power, symmetric power and divided power functors.

Let $\mathcal{P}$ be an additive category, $\mathcal{M}$ an abelian category, and $F: \mathcal{P} \rightarrow \mathcal{M}$ a functor with $F(0)=0$.

Definition 1.1. Let $k \geq 0$. For any $V_{1}, \ldots, V_{k} \in \mathcal{P}$ and $1 \leq i \leq k$, let

$$
p_{i}: V_{1} \oplus \ldots \oplus V_{k} \rightarrow V_{i} \rightarrow V_{1} \oplus \ldots \oplus V_{k}
$$

denote the $i$-th projection. The $k$-functor

$$
\begin{aligned}
& \operatorname{cr}_{k}(F): \quad \mathcal{P}^{k} \quad \rightarrow \mathcal{M} \\
& \left(V_{1}, \ldots, V_{k}\right) \mapsto \operatorname{Im}\left(\sum_{j=1}^{k}(-1)^{k-j} \sum_{1 \leq i_{1}<\ldots<i_{j} \leq k} F\left(p_{i_{1}}+\ldots+p_{i_{j}}\right)\right)
\end{aligned}
$$

is called the $k$-th cross effect of $F$; here, for any $V_{1}, \ldots, V_{k}, W_{1}, \ldots, W_{k} \in \mathcal{P}$ and $f_{1} \in \operatorname{Hom}_{\mathcal{P}}\left(V_{1}, W_{1}\right), \ldots, f_{k} \in \operatorname{Hom}_{\mathcal{P}}\left(V_{k}, W_{k}\right)$, the map

$$
\operatorname{cr}_{k}(F)\left(f_{1}, \ldots, f_{k}\right): \mathrm{cr}_{k}(F)\left(V_{1}, \ldots, V_{k}\right) \rightarrow \mathrm{cr}_{k}(F)\left(W_{1}, \ldots, W_{k}\right)
$$

is induced by $f_{1} \oplus \ldots \oplus f_{k} \in \operatorname{Hom}_{\mathcal{P}}\left(V_{1} \oplus \ldots \oplus V_{k}, W_{1} \oplus \ldots \oplus W_{k}\right)$. $F$ is said to be a functor of degree $\leq k$ if $\operatorname{cr}_{k+1}(F)$ is identically zero.

The cross effect functors $\mathrm{cr}_{k}(F), k \geq 0$, have the following properties. We obviously have $\operatorname{cr}_{0}(F) \equiv 0$ and $\operatorname{cr}_{1}(F)=F$. Furthermore, we have $\operatorname{cr}_{k}(F)\left(V_{1}, \ldots, V_{k}\right)=$ 0 , if $V_{i}=0$ for one $i \in\{1, \ldots, k\}$ (see Theorem 9.2 on p. 79 in [EM]). The canonical action of the symmetric group $\Sigma_{k}$ on $V^{k}=V \oplus \ldots \oplus V$ induces a natural action of $\Sigma_{k}$ on $\operatorname{cr}_{k}(F)(V, \ldots, V)$ (see Theorem 9.3 on p. 80 in [EM]). Any natural transformation $F \rightarrow G$ between functors $F$ and $G$ from $\mathcal{P}$ to $\mathcal{M}$ induces a natural transformation $\operatorname{cr}_{k}(F) \rightarrow \operatorname{cr}_{k}(G)$ (see Theorem 9.5 on p. 80 in [EM]). The most important property is the following proposition.

Proposition 1.2. For any $k, l \geq 1$ and $V_{1}, \ldots, V_{l} \in \mathcal{P}$, we have a canonical isomorphism

$$
\begin{aligned}
& \operatorname{cr}_{k}(F)\left(\ldots, V_{1} \oplus \ldots \oplus V_{l}, \ldots\right) \\
& \quad \cong \bigoplus_{1 \leq j \leq l} \bigoplus_{1 \leq i_{1}<\ldots<i_{j} \leq l} \operatorname{cr}_{k+j}(F)\left(\ldots, V_{i_{1}}, \ldots, V_{i_{j}}, \ldots\right)
\end{aligned}
$$

which is functorial in $V_{1}, \ldots, V_{l}$. In particular, $F$ is of degree $\leq k$, if and only if $\mathrm{cr}_{k}(F)$ is a $k$-additive functor.

Proof. See Theorem 9.1, Lemma 9.8 and Lemma 9.9 in [EM].

Proposition 1.2 states that

$$
F\left(V_{1} \oplus V_{2}\right) \cong F\left(V_{1}\right) \oplus F\left(V_{2}\right) \oplus \mathrm{cr}_{2}(F)\left(V_{1}, V_{2}\right)
$$


for all $V_{1}, V_{2} \in \mathcal{P}$; i.e., $\mathrm{cr}_{2}(F)$ measures the deviation from additivity of the functor $F$. This isomorphism can also be used to define $\mathrm{cr}_{2}(F)$ (see section 3 in [JM]). Similarly, the higher cross effects can inductively be defined by the isomorphism

$$
\begin{aligned}
& \quad \mathrm{cr}_{k}(F)\left(V_{1}, \ldots, V_{k-1}, V_{k} \oplus V_{k+1}\right) \\
& \cong \quad \operatorname{cr}_{k}(F)\left(V_{1}, \ldots, V_{k-1}, V_{k}\right) \oplus \mathrm{cr}_{k}(F)\left(V_{1}, \ldots, V_{k-1}, V_{k+1}\right) \\
& \quad \oplus \mathrm{cr}_{k+1}(F)\left(V_{1}, \ldots, V_{k}, V_{k+1}\right)
\end{aligned}
$$

(see section 7 in $[\mathrm{JM}]$ ). In fact, we will use this definition whenever we have to compute cross effect functors.

Definition 1.3. Let $l \geq k \geq 1, \varepsilon=\left(\varepsilon_{1}, \ldots, \varepsilon_{k}\right) \in\{1, \ldots, l\}^{k}$ with $|\varepsilon|=\varepsilon_{1}+\ldots+$ $\varepsilon_{k}=l$ and $V_{1}, \ldots, V_{k} \in \mathcal{P}$. The composition

$$
\begin{aligned}
\Delta_{\varepsilon}: \mathrm{cr}_{k}(F)\left(V_{1}, \ldots, V_{k}\right) \stackrel{\operatorname{cr}_{k}(F)(\Delta, \ldots, \Delta)}{\longrightarrow} \operatorname{cr}_{k}(F)\left(V_{1}^{\varepsilon_{1}}, \ldots, V_{k}^{\varepsilon_{k}}\right) \\
\stackrel{\pi}{\longrightarrow} \operatorname{cr}_{l}(F)\left(V_{1}, \ldots, V_{1}, \ldots, V_{k}, \ldots, V_{k}\right)
\end{aligned}
$$

of the map $\operatorname{cr}_{k}(F)(\Delta, \ldots, \Delta)$ (induced by the diagonal maps $\Delta: V_{i} \rightarrow V_{i}^{\varepsilon_{i}}, i=$ $1, \ldots, k)$ with the canonical projection $\pi$ (according to Proposition 1.2) is called diagonal map associated with $\varepsilon$. The analogous composition

$$
\begin{array}{r}
+_{\varepsilon}: \operatorname{cr}_{l}(F)\left(V_{1}, \ldots, V_{1}, \ldots, V_{k}, \ldots, V_{k}\right) \longleftrightarrow \operatorname{cr}_{k}(F)\left(V_{1}^{\varepsilon_{1}}, \ldots, V_{k}^{\varepsilon_{k}}\right) \\
\stackrel{\operatorname{cr}_{k}(F)(+, \ldots,+)}{\longrightarrow} \operatorname{cr}_{k}(F)\left(V_{1}, \ldots, V_{k}\right)
\end{array}
$$

is called plus map associated with $\varepsilon$.

Obviously, the maps $\Delta_{\varepsilon}$ and $+_{\varepsilon}$ form natural transformations between the functors $\operatorname{cr}_{k}(F)$ and $\operatorname{cr}_{l}(F) \circ\left(\Delta_{\varepsilon_{1}}, \ldots, \Delta_{\varepsilon_{k}}\right)$ from $\mathcal{P}^{k}$ to $\mathcal{M}$. One easily sees that the map $\Delta_{\varepsilon}$ can be decomposed into a composition of maps $\Delta_{\delta}$ with $\delta \in\{1,2\}^{j}$ such that $|\delta|=j+1$ and $j \in\{k, \ldots, l-1\}$. The same holds for $+_{\varepsilon}$.

Example 1.4. Let $\mathcal{P}=\mathcal{M}$ be the category of modules over a commutative ring $A$. For any $n \geq 1$, let $\operatorname{Sym}^{n}: \mathcal{P} \rightarrow \mathcal{M}$ denote the $n$-th symmetric power functor.

(a) For all $k \geq 1$ and $V_{1}, \ldots, V_{k} \in \mathcal{P}$, we have

$$
\operatorname{cr}_{k}\left(\operatorname{Sym}^{n}\right)\left(V_{1}, \ldots, V_{k}\right) \cong \bigoplus_{\substack{\left(n_{1}, \ldots, n_{k}\right) \in\{1, \ldots, n\}^{k} \\ n_{1}+\ldots+n_{k}=n}} \operatorname{Sym}^{n_{1}}\left(V_{1}\right) \otimes \ldots \otimes \operatorname{Sym}^{n_{k}}\left(V_{k}\right) .
$$

(b) Let $n=2$. Then the following diagrams commute for all $V \in \mathcal{P}$ :
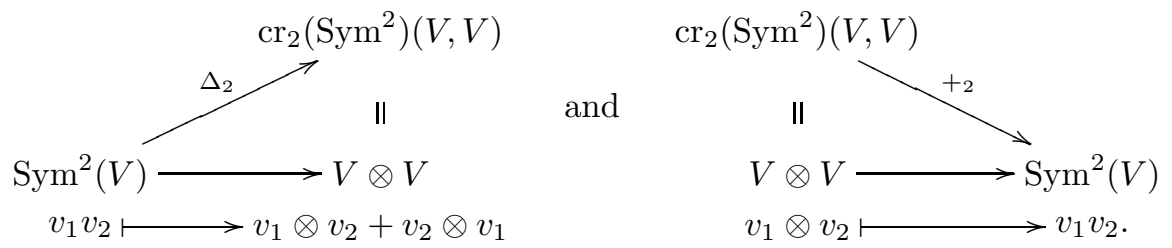

(c) Let $n=3$. By virtue of the isomorphisms given in (a), the diagonal and plus maps can be described as follows (for $V, W \in \mathcal{P}$ ):

$$
\begin{array}{rlc}
\Delta_{3}: \operatorname{Sym}^{3}(V) & \rightarrow & V \otimes V \otimes V \\
v_{1} v_{2} v_{3} & \mapsto \sum_{\sigma \in \Sigma_{3}} v_{\sigma(1)} \otimes v_{\sigma(2)} \otimes v_{\sigma(3)} .
\end{array}
$$




$$
\begin{aligned}
& \Delta_{(2,1)}: \quad \operatorname{Sym}^{2}(V) \otimes W \oplus V \otimes \operatorname{Sym}^{2}(W) \quad \rightarrow \quad V \otimes V \otimes W \\
& \left(v_{1} v_{2} \otimes w, v \otimes w_{1} w_{2}\right) \quad \mapsto v_{1} \otimes v_{2} \otimes w+v_{2} \otimes v_{1} \otimes w . \\
& \Delta_{(1,2)}: \operatorname{Sym}^{2}(V) \otimes W \oplus V \otimes \operatorname{Sym}^{2}(W) \quad \rightarrow \quad V \otimes W \otimes W \\
& \left(v_{1} v_{2} \otimes w, v \otimes w_{1} w_{2}\right) \quad \mapsto v \otimes w_{1} \otimes w_{2}+v \otimes w_{2} \otimes w_{1} . \\
& \Delta_{2}: \operatorname{Sym}^{3}(V) \rightarrow \operatorname{Sym}^{2}(V) \otimes V \oplus V \otimes \operatorname{Sym}^{2}(V) \\
& v_{1} v_{2} v_{3} \mapsto\left(v_{2} v_{3} \otimes v_{1}+v_{1} v_{3} \otimes v_{2}+v_{1} v_{2} \otimes v_{3}, v_{1} \otimes v_{2} v_{3}+v_{2} \otimes v_{1} v_{3}+v_{3} \otimes v_{1} v_{2}\right) \text {. } \\
& +_{3}: \quad V \otimes V \otimes V \rightarrow \operatorname{Sym}^{3}(V) \\
& v_{1} \otimes v_{2} \otimes v_{3} \quad \mapsto \quad v_{1} v_{2} v_{3} \\
& +_{(2,1)}: \quad V \otimes V \otimes W \rightarrow \operatorname{Sym}^{2}(V) \otimes W \oplus V \otimes \operatorname{Sym}^{2}(W), \\
& v_{1} \otimes v_{2} \otimes w \quad \mapsto \quad\left(v_{1} v_{2} \otimes w, 0\right) . \\
& +_{(1,2)}: \quad V \otimes W \otimes W \quad \rightarrow \quad \operatorname{Sym}^{2}(V) \otimes W \oplus V \otimes \operatorname{Sym}^{2}(W) \\
& v \otimes w_{1} \otimes w_{2} \quad \mapsto \quad\left(0, v \otimes w_{1} w_{2}\right) . \\
& +_{2}: \operatorname{Sym}^{2}(V) \otimes V \oplus V \otimes \operatorname{Sym}^{2}(V) \rightarrow \operatorname{Sym}^{3}(V) \\
& \left(v_{1} v_{2} \otimes v_{3}, v_{1}^{\prime} \otimes v_{2}^{\prime} v_{3}^{\prime}\right) \quad \mapsto v_{1} v_{2} v_{3}+v_{1}^{\prime} v_{2}^{\prime} v_{3}^{\prime} .
\end{aligned}
$$

Proof. Straightforward.

The following theorem states that any functor $F$ of finite degree from the category of all $\mathrm{f}$. g. projective modules over a ring $A$ to any abelian category $\mathcal{M}$ is determined by the objects $\operatorname{cr}_{i}(F)(A, \ldots, A), i \geq 0$, the diagonal and plus maps between these objects, and the actions

$$
A \rightarrow \operatorname{End}_{\mathcal{M}}\left(\operatorname{cr}_{i}(F)(A, \ldots, A)\right), \quad a \mapsto \operatorname{cr}_{i}(F)(1, \ldots, 1, a, 1, \ldots, 1),
$$

of the multiplicative monoid $A$ on these objects. (Here, 1 and $a$ denote the multiplication with 1 and $a$ on $A$, respectively.)

Theorem 1.5. Let $A$ be a ring, $\mathcal{M}$ an abelian category, $d \in \mathbb{N}$, and

$$
F, G:(f . g \text {. projective A-modules }) \Longrightarrow \mathcal{M}
$$

two functors of degree $\leq d$ with $F(0)=0=G(0)$. Suppose that there exist isomorphisms

$$
\alpha_{i}(A, \ldots, A): \operatorname{cr}_{i}(F)(A, \ldots, A) \stackrel{\sim}{\longrightarrow} \operatorname{cr}_{i}(G)(A, \ldots, A), \quad i=1, \ldots, d,
$$

which are compatible with the action of $A$ in each component and which make the following diagrams commute for all $i \in\{1, \ldots, d-1\}$ and $\varepsilon \in\{1,2\}^{i}$ with $|\varepsilon|=i+1$ :

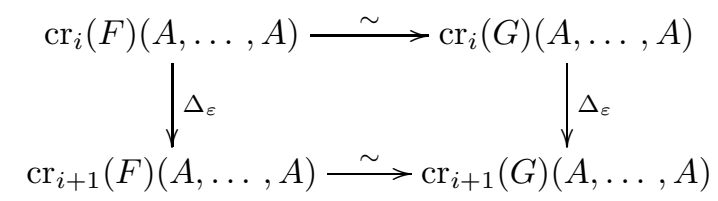

and

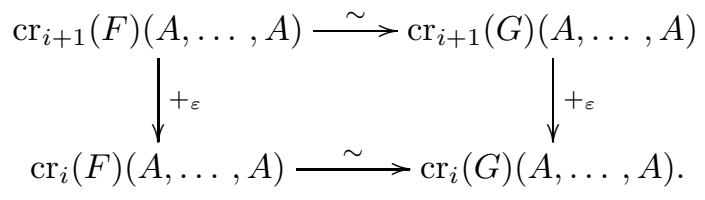

Then the functors $F$ and $G$ are isomorphic. 
Proof. For any $i \geq 1$ and $n_{1}, \ldots, n_{i} \geq 1$, we write $\operatorname{cr}_{i}(F)\left(A^{n_{1}}, \ldots, A^{n_{i}}\right)$ as a direct sum of copies of $\mathrm{cr}_{i}(F)(A, \ldots, A), \ldots, \mathrm{cr}_{d}(F)(A, \ldots, A)$ according to Proposition 1.2 ; in the same way, we write $\operatorname{cr}_{i}(G)\left(A^{n_{1}}, \ldots, A^{n_{i}}\right)$ as a direct sum of copies of $\mathrm{cr}_{i}(G)(A, \ldots, A), \ldots, \mathrm{cr}_{d}(G)(A, \ldots, A)$. The corresponding sum of copies of the isomorphisms $\alpha_{i}(A, \ldots, A), \ldots, \alpha_{d}(A, \ldots, A)$ then yields an isomorphism

$$
\alpha_{i}\left(A^{n_{1}}, \ldots, A^{n_{i}}\right): \operatorname{cr}_{i}(F)\left(A^{n_{1}}, \ldots, A^{n_{i}}\right) \stackrel{\sim}{\longrightarrow} \operatorname{cr}_{i}(G)\left(A^{n_{1}}, \ldots, A^{n_{i}}\right) .
$$

Now, we show by descending induction on $i$ that, for all $m_{1}, \ldots, m_{i}, n_{1}, \ldots, n_{i} \geq 1$ and for all matrices $\mathcal{A}_{1} \in \operatorname{Hom}\left(A^{n_{1}}, A^{m_{1}}\right), \ldots, \mathcal{A}_{i} \in \operatorname{Hom}\left(A^{n_{i}}, A^{m_{i}}\right)$, the following diagram commutes:

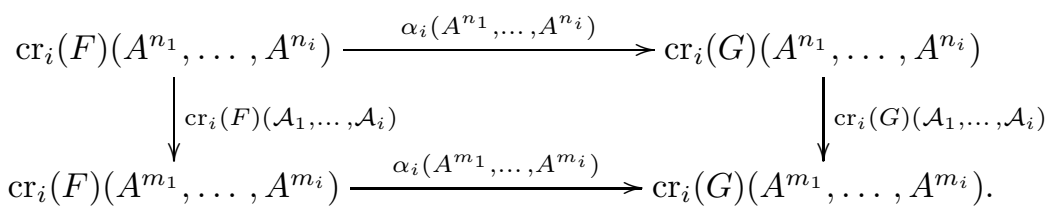

For $i=d+1$, this assertion is clear since $\mathrm{cr}_{d+1}(F)$ and $\mathrm{cr}_{d+1}(G)$ are identically zero. So, we may assume that $i \leq d$ and that the assertion is already proved for $i+1$. Writing $\left(\mathcal{A}_{1}, \ldots, \mathcal{A}_{i}\right)$ as the composition of $\left(\mathcal{A}_{1}, \mathrm{id}, \ldots, \mathrm{id}\right), \ldots,\left(\mathrm{id}, \ldots, \mathrm{id}, \mathcal{A}_{i}\right)$ and using symmetry, we may furthermore assume that $\mathcal{A}_{2}, \ldots, \mathcal{A}_{i}$ are the identity matrices. By construction of $\alpha_{i}\left(A^{n_{1}}, \ldots, A^{n_{i}}\right)$, we may finally assume that $n_{2}=$ $\ldots=n_{i}=1$. Now, we write $\mathcal{A}$ for $\mathcal{A}_{1}, \operatorname{cr}_{i}(F)\left(A^{n}\right)$ for $\operatorname{cr}_{i}(F)\left(A^{n_{1}}, A, \ldots, A\right)$, and similarly for $\operatorname{cr}_{i}(G)\left(A^{n_{1}}, A, \ldots, A\right)$, etc. By construction, the above diagram commutes, if $\mathcal{A}$ is a standard projection $A^{n} \rightarrow A$ or a standard embedding $A \rightarrow A^{m}$. Furthermore, the diagram commutes if $n=m=1$ by assumption. Hence, the diagram commutes for all matrices $\mathcal{A}$ which have at most one entry which is different from zero. Thus it suffices to show that the diagram commutes for the matrix $\mathcal{A}+\mathcal{B}$ if it commutes for the matrices $\mathcal{A}$ and $\mathcal{B}$. For this, we decompose $\mathcal{A}+\mathcal{B}$ into the composition

$$
A^{n} \stackrel{\Delta}{\longrightarrow} A^{n} \oplus A^{n} \stackrel{\mathcal{A} \oplus \mathcal{B}}{\longrightarrow} A^{m} \oplus A^{m} \stackrel{+}{\longrightarrow} A^{m}
$$

and identify $\operatorname{cr}_{i}(-)\left(A^{n} \oplus A^{n}\right)$ with $\operatorname{cr}_{i}(-)\left(A^{n}\right) \oplus \mathrm{cr}_{i}\left(A^{n}\right) \oplus \mathrm{cr}_{i+1}(-)\left(A^{n}, A^{n}\right)$ according to Proposition 1.2. By construction and assumption, the following diagram commutes:

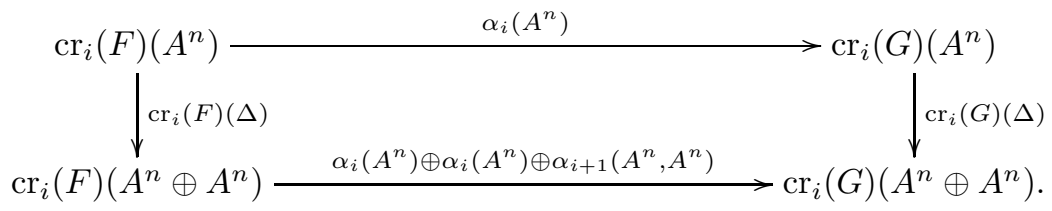

(To see this, one decomposes the map $\mathrm{cr}_{i}(F)(\Delta)$ into a direct sum of copies of the maps

$\mathrm{cr}_{i+j}(F)(\Delta, \ldots, \Delta): \mathrm{cr}_{i+j}(F)(A, \ldots, A) \rightarrow \mathrm{cr}_{i+j}(F)(A \oplus A, \ldots, A \oplus A), \quad j \geq 0$, according to Proposition 1.2. The composition of such a map with a natural projection $\mathrm{cr}_{i+j}(F)(A \oplus A, \ldots, A \oplus A) \rightarrow \mathrm{cr}_{i+j+k}(F)(A, \ldots, A)$ (again according to Proposition 1.2) is then a diagonal map $\Delta_{\varepsilon}$ for some $\varepsilon \in\{1,2\}^{i+j}$ with $|\varepsilon|=i+j+k$.) Likewise, the corresponding diagram for + in place of $\Delta$ commutes. 
By the assumption on $\mathcal{A}$ and $\mathcal{B}$ and by the induction hypothesis, the following diagram also commutes:

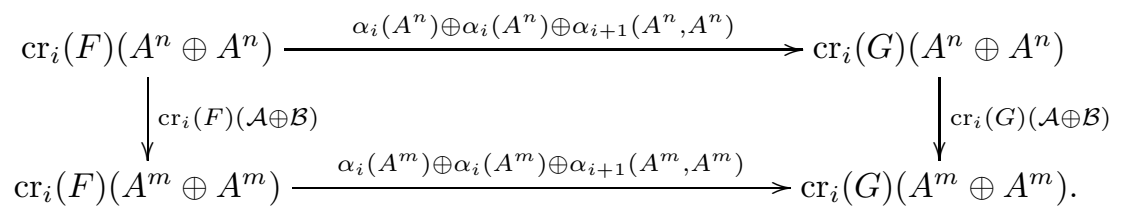

Now, the proof of the above assertion is complete.

This assertion implies in particular that, for all $n \geq 1$ and for all projectors $\mathcal{A} \in$ $\operatorname{End}\left(A^{n}\right)$, the isomorphism

$$
F\left(A^{n}\right)=\operatorname{cr}_{1}(F)\left(A^{n}\right) \stackrel{\alpha_{1}\left(A^{n}\right)}{\longrightarrow} \operatorname{cr}_{1}(G)\left(A^{n}\right)=G\left(A^{n}\right)
$$

induces an isomorphism

$$
\alpha_{1}(\operatorname{Im}(\mathcal{A})): F(\operatorname{Im}(\mathcal{A})) \cong \operatorname{Im}(F(\mathcal{A})) \stackrel{\sim}{\longrightarrow} \operatorname{Im}(G(\mathcal{A})) \cong G(\operatorname{Im}(\mathcal{A})) .
$$

If $\mathcal{B} \in \operatorname{End}\left(A^{m}\right)$ is a further projector and $f: \operatorname{Im}(\mathcal{A}) \rightarrow \operatorname{Im}(\mathcal{B})$ is an $A$-homomorphism, then the diagram

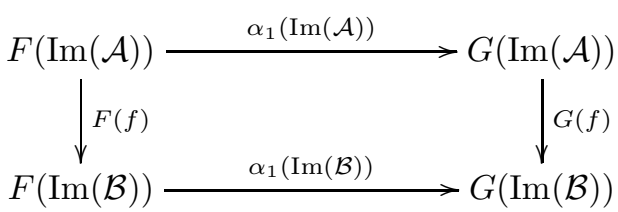

commutes (again by the above assertion). Finally, if $V$ is an arbitrary f. g. projective $A$-module, we choose a projector $\mathcal{A}$ as above and an isomorphism $V \cong \operatorname{Im}(\mathcal{A})$ and define $\alpha_{1}(V)$ to be the composition

$$
F(V) \cong F(\operatorname{Im}(\mathcal{A})) \stackrel{\alpha_{1}(\operatorname{Im}(\mathcal{A}))}{\longrightarrow} G(\operatorname{Im}(\mathcal{A})) \cong G(V) .
$$

It is clear that $\alpha_{1}(V)$ does not depend on the chosen projector and on the chosen isomorphism and that $\alpha_{1}$ is an isomorphism of functors. This proves Theorem 1.5 .

Corollary 1.6 (Characterization of the exterior power functors). Let $A$ be a commutative ring, $B$ a further ring, $d \in \mathbb{N}$, and

$$
F: \mathcal{P}_{A}:=(f . g \text {. projective } A \text {-modules }) \rightarrow(B \text {-modules })=: \mathcal{M}_{B}
$$

a functor with $F(0)=0$ and with the following properties:

(i) $\operatorname{cr}_{d}(F)$ is d-additive, i.e., $\operatorname{cr}_{d+1}(F) \equiv 0$.

(ii) The $d$ A-module structures on $\mathrm{cr}_{d}(F)(A, \ldots, A)$ coincide.

(iii) $F\left(A^{d-1}\right)=0$.

Then we have for all $V \in \mathcal{P}_{A}$,

$$
F(V) \cong \Lambda^{d}(V) \otimes_{A} \mathrm{cr}_{d}(F)(A, \ldots, A) .
$$

Proof. The assumption $F\left(A^{d-1}\right)=0$ implies

$$
\operatorname{cr}_{1}(F)(A)=0, \ldots, \operatorname{cr}_{d-1}(F)(A, \ldots, A)=0
$$


by Proposition 1.2. Similarly, for the functor $G$ defined by $G(V):=\Lambda^{d}(V) \otimes_{A}$ $\mathrm{cr}_{d}(F)(A, \ldots, A)$, we have $\mathrm{cr}_{1}(G)(A)=0, \ldots, \mathrm{cr}_{d-1}(G)(A, \ldots, A)=0$. Furthermore, we have $\mathrm{cr}_{d}(G)(A, \ldots, A) \cong \mathrm{cr}_{d}(F)(A, \ldots, A)$. Thus, Corollary 1.6 follows from Theorem 1.5.

Example 1.7. In the case $d=1$, Corollary 1.6 yields the following statement which has already been proved in $[\mathrm{E}]$ and $\left[\mathrm{Wa}\right.$ : Each additive functor $F: \mathcal{P}_{A} \rightarrow \mathcal{M}_{B}$ is isomorphic to the tensor functor $-\otimes F(A)$. By induction, we obtain the following generalization: Let $F: \mathcal{P}_{A}^{d} \rightarrow \mathcal{M}$ be a $d$-additive functor such that the $d A$-module structures on $F(A, \ldots, A)$ coincide. Then we have for all $V_{1}, \ldots, V_{d} \in \mathcal{P}_{A}$,

$$
F\left(V_{1}, \ldots, V_{d}\right) \cong V_{1} \otimes_{A} \ldots \otimes_{A} V_{d} \otimes_{A} F(A, \ldots, A) .
$$

Proposition 1.8 (Characterization of the symmetric power functors). Let $A, B$, $d$, and $F$ be as in Corollary 1.6. In contrast to Corollary 1.6, we assume however that $F$ has the following property in place of property (iii): For all $i=1, \ldots, d-1$, the B-module homomorphism

$$
\operatorname{plus}_{i}: \bigoplus_{\varepsilon \in\{1, \ldots, d\}^{i},|\varepsilon|=d} \operatorname{cr}_{d}(F)(A, \ldots, A) \stackrel{(+\varepsilon)_{\varepsilon \in\{1, \ldots, d\}^{i},|\varepsilon|=d}}{\longrightarrow} \operatorname{cr}_{i}(F)(A, \ldots, A)
$$

is bijective. Then we have for all $V \in \mathcal{P}_{A}$,

$$
F(V) \cong \operatorname{Sym}^{d}(V) \otimes_{A} \mathrm{cr}_{d}(F)(A, \ldots, A) .
$$

Proof. We view $\mathrm{cr}_{d}(F)(V, \ldots, V)$ as a $\Sigma_{d}$-module with the action introduced above and $F(V)$ as a $\Sigma_{d}$-module with trivial action. Then, the plus map

$$
+_{d}: \mathrm{cr}_{d}(F)(V, \ldots, V) \rightarrow F(V)
$$

is obviously compatible with these $\Sigma_{d}$-actions. Since $+_{d}: \operatorname{cr}_{d}(F)(A, \ldots, A) \rightarrow$ $F(A)$ is bijective by assumption, the symmetric group $\Sigma_{d}$ acts trivially on the object $\mathrm{cr}_{d}(F)(A, \ldots, A)$. Hence, the composition

$$
V^{\otimes d} \otimes_{A} \mathrm{cr}_{d}(F)(A, \ldots, A) \cong \mathrm{cr}_{d}(F)(V, \ldots, V) \stackrel{+_{d}}{\longrightarrow} F(V)
$$

of the isomorphism given in Example 1.7 with the plus map $+_{d}$ induces a $B$-module homomorphism

$$
\alpha(V): \operatorname{Sym}^{d}(V) \otimes_{A} \mathrm{cr}_{d}(F)(A, \ldots, A) \rightarrow F(V)
$$

which is functorial in $V$. One easily proves that, for all $i=1, \ldots, d$, the homomorphism $\operatorname{cr}_{i}(\alpha)(A, \ldots, A)$ coincides with the isomorphism plus $i$ by virtue of the isomorphism

$$
\operatorname{cr}_{i}\left(\operatorname{Sym}^{d}\right)(A, \ldots, A) \cong \bigoplus_{\varepsilon \in\{1, \ldots, d\}^{i},|\varepsilon|=d} A
$$

(see Example 1.4(a)). Hence, $\mathrm{cr}_{i}(\alpha)(A, \ldots, A)$ is bijective. Then, by Proposition $1.2, \alpha(V)$ is bijective for all f. g. free $A$-modules $V$. Again by Proposition 1.2, the same then holds for all $V \in \mathcal{P}_{A}$. This proves Proposition 1.8.

Proposition 1.9 (Characterization of the divided power functors). Let $A, B, d$, and $F$ be as in Corollary 1.6. In contrast to Corollary 1.6, we assume however that 
$F$ has the following property in place of property (iii): For all $i=1, \ldots, d-1$, the B-module homomorphism

$$
\operatorname{diag}_{i}: \operatorname{cr}_{i}(F)(A, \ldots, A) \stackrel{\left(\Delta_{\varepsilon}\right)_{\varepsilon \in\{1, \ldots, d\}^{i},|\varepsilon|=d}}{\longrightarrow} \bigoplus_{\varepsilon \in\{1, \ldots, d\}^{i},|\varepsilon|=d} \operatorname{cr}_{d}(F)(A, \ldots, A)
$$

is bijective. Then we have for all $V \in \mathcal{P}_{A}$,

$$
F(V) \cong D^{d}(V) \otimes_{A} \operatorname{cr}_{d}(F)(A, \ldots, A) .
$$

Proof. The $d$-th divided power $D^{d}(V)$ of an f. g. projective $A$-module $V$ is the module of fixed elements under the natural action of the symmetric group $\Sigma_{d}$ on $V^{\otimes d}$. Thus, Proposition 1.9 can be proved analogously to Proposition 1.8.

Remark 1.10. For any functor $F: \mathcal{P}_{A} \rightarrow \mathcal{M}_{B}$ with $F(0)=0$, the composition $F(A) \stackrel{\Delta_{2}}{\longrightarrow} \operatorname{cr}_{2}(F)(A, A) \stackrel{+_{2}}{\longrightarrow} F(A)$ equals $F\left(2 \cdot \mathrm{id}_{A}\right)-2 \cdot \mathrm{id}_{F(A)}$. Now assume that $d=2$. Under the assumptions of Proposition 1.8, the endomorphism $F\left(2 \cdot \operatorname{id}_{A}\right)$ of $F(A)$ then corresponds to the endomorphism $\mathrm{cr}_{2}(F)\left(2 \cdot \mathrm{id}_{A}, 2 \cdot \mathrm{id}_{A}\right)=4 \cdot$ id of $\operatorname{cr}_{2}(F)(A, A)$; hence, $F\left(2 \cdot \operatorname{id}_{A}\right)=4 \cdot \operatorname{id}_{F(A)}$. Thus, $+_{2} \circ \Delta_{2}=2 \cdot \operatorname{id}_{F(A)}$. This proves that the assumptions of Proposition 1.8 also determine the map $\Delta_{2}$. Thus, Proposition 1.8 follows from Theorem 1.5 in the case $d=2$. The same holds for Proposition 1.9. It seems that a simple generalization of this argument to the case $d \geq 2$ does not exist.

\section{Simplicial modules AND Koszul COMPlexes}

First, we recall the Dold-Kan correspondence; in particular, we introduce the functors $\Gamma$ and $N$ between the category of simpicial modules and the category of complexes. Then, we recall the definition of the $n$-th Koszul complex $\operatorname{Kos}^{n}(f)$ associated with a homomorphism $f: P \rightarrow Q$ between the projective modules $P, Q$ over a commutative ring $R$. Finally, we construct an explicit complex homomorphism $u^{n}(f)$ from $\operatorname{Kos}^{n}(f)$ to $N \operatorname{Sym}^{n} \Gamma(\ldots \rightarrow P \stackrel{f}{\rightarrow} Q)$ and prove that $u^{n}(f)$ is a quasi-isomorphism.

Let $\mathcal{M}$ be an additive category. Let $\Delta$ denote the category whose objects are the sets $[n]=\{0<1<\ldots<n\}, n \geq 0$, and whose morphisms are the order preserving set maps. For $0 \leq i \leq n+1$, let $\delta_{i}^{n}:[n] \rightarrow[n+1]$ denote the injective order preserving map given by $\operatorname{Im}\left(\delta_{i}^{n}\right)=[n+1] \backslash\{i\}$. For $0 \leq i \leq n$, let $\sigma_{i}^{n}:[n+1] \rightarrow[n]$ denote the surjective order preserving map given by $\left(\sigma_{i}^{n}\right)^{-1}(i)=\{i, i+1\}$. A simplicial object $X$. in $\mathcal{M}$ is a contravariant functor from $\Delta$ to $\mathcal{M}$. We write $X_{n}$ for $X$.([n]), $d_{i}: X_{n+1} \rightarrow X_{n}$ for $X .\left(\delta_{i}^{n}\right)$ and $s_{i}: X_{n} \rightarrow X_{n+1}$ for $X .\left(\sigma_{i}^{n}\right)$. A simplicial homomorphism $f .: X$. $\rightarrow Y$. between two simplicial objects $X$., $Y$. in $\mathcal{M}$ is a natural transformation. A simplicial homotopy between simplicial homomorphisms $f, g$ : $X . \Longrightarrow Y$. consists of homomorphisms $h_{i}(n): X_{n} \rightarrow Y_{n+1}, n \geq 0,0 \leq i \leq n$, such that $d_{0} h_{0}(n)=f_{n}$ and $d_{n+1} h_{n}(n)=g_{n}$ for all $n \geq 0$ and such that certain further relations $d_{i} h_{j}(n)=\ldots$ and $s_{i} h_{j}(n)=\ldots$ hold (see 8.3.11 in W] or $(2.3)$ in [JM]). A complex $K$. in $\mathcal{M}$ is a contravariant functor from the ordered set $\mathbb{N}_{0}$ to the category $\mathcal{M}$ such that $\partial^{2}=0$ where $\partial: K_{i+1} \rightarrow K_{i}$ denotes the differential. The normalized complex $N(X$.) of a simplicial object $X$. in an abelian category $\mathcal{M}$ is given by

$$
N(X .)_{n}:=X_{n} / \sum_{i=0}^{n-1} \operatorname{Im}\left(s_{i}: X_{n-1} \rightarrow X_{n}\right)
$$


the differential in $N(X$.) is

$$
\partial=\sum_{i=0}^{n}(-1)^{i} d_{i}: N(X .)_{n} \rightarrow N(X .)_{n-1}
$$

(for all $n \geq 0$ ). Thus, we have a functor

$$
N: \operatorname{Simp}(\mathcal{M}) \rightarrow \operatorname{Compl}(\mathcal{M})
$$

from the category $\operatorname{Simp}(\mathcal{M})$ of simplicial objects in $\mathcal{M}$ to the category $\operatorname{Compl}(\mathcal{M})$ of complexes in $\mathcal{M}$. We define a functor

$$
\Gamma: \operatorname{Compl}(\mathcal{M}) \rightarrow \operatorname{Simp}(\mathcal{M})
$$

in the reverse direction as follows. For $n \geq k$, let $\operatorname{Sur}([n],[k])$ denote the set of surjective order preserving maps from $[n]$ to $[k]$. For $(M ., \partial) \in \operatorname{Compl}(\mathcal{M})$ and $n \geq 0$, let $\Gamma(M .)_{n}$ be defined by

$$
\Gamma(M .)_{n}:=\bigoplus_{k=0}^{n} \bigoplus_{\sigma \in \operatorname{Sur}([n],[k])} M_{k} .
$$

Furthermore, $d_{i}: \Gamma\left(M_{.}\right)_{n} \rightarrow \Gamma(M .)_{n-1}, i \in\{0, \ldots, n\}$, and $s_{i}: \Gamma(M .)_{n} \rightarrow$ $\Gamma(M .)_{n+1}, i \in\{0, \ldots, n\}$, are defined by

$$
\begin{aligned}
& d_{i}(m, \sigma):= \begin{cases}\left(m, \sigma \delta_{i}\right) & \text { if } \sigma \delta_{i} \in \operatorname{Sur}([n-1],[k]), \\
(\partial(m), \hat{\sigma}) & \text { if } \sigma \delta_{i}=\delta_{0} \hat{\sigma}(\text { where } \hat{\sigma} \in \operatorname{Sur}([n-1],[k-1])), \\
0 & \text { if } \sigma \delta_{i}=\delta_{j} \hat{\sigma} \text { with } j \geq 1,\end{cases} \\
& s_{i}(m, \sigma):=\left(m, \sigma \sigma_{i}\right)
\end{aligned}
$$

(for $k \in\{0, \ldots, n\}$ and $\left.(m, \sigma) \in M_{k} \times \operatorname{Sur}([n],[k])\right)$. One easily verifies that these definitions determine a simplicial object $\Gamma(M$.) in $\mathcal{M}$ (cf. section 2 in [JM] or section 8.4 in $[\mathrm{W}]$ ).

Proposition 2.1 (Dold-Kan correspondence). For any abelian category $\mathcal{M}$, the functors $N$ and $\Gamma$ are inverse to each other (up to canonical isomorphisms). A simplicial homotopy between two simplicial homomorphisms induces a homotopy between the corresponding complex homomorphisms and vice versa.

Proof. See section 8.4 in [W].

Now, let $F: \mathcal{P} \rightarrow \mathcal{M}$ be a functor from an additive category $\mathcal{P}$ to an abelian category $\mathcal{M}$ with $F(0)=0$. If $F$ is additive, then the induced functor $\operatorname{Compl}(\mathcal{P}) \rightarrow$ $\operatorname{Compl}(\mathcal{M}), K$. $F \circ K$., maps homotopic complex homomorphisms to homotopic complex homomorphisms. In the simplicial world, the analogous statement holds even for an arbitrary functor $F$ : The induced functor $\operatorname{Simp}(\mathcal{P}) \rightarrow \operatorname{Simp}(\mathcal{M}), X$. $F X$. : $=F \circ X$., maps simplicial homotopies to simplicial homotopies since only compositions (and no sums) are involved in the defining relations of a simplicial homotopy. Hence, using the Dold-Kan correspondence, we obtain a functor

$$
\operatorname{Compl}(\mathcal{P}) \rightarrow \operatorname{Compl}(\mathcal{M}), \quad K . \mapsto N F \Gamma(K .),
$$

which maps homotopic complex homomorphisms to homotopic complex homomorphisms and which is obviously isomorphic to the functor $K$. $\mapsto F \circ K$., if $F$ is additive. (This basic fact has already been observed by Dold and Puppe in $[\overline{D P}$.) If $K$. is a complex in $\mathcal{P}$ with $K_{n}=0$ for all $n \geq 2$, then the complex $N F \Gamma(K$.) can be described as follows by using the cross effect functors introduced in section 1 . 
Lemma 2.2. Let $f: P \rightarrow Q$ be a homomorphism in $\mathcal{P}$. We consider $f$ as a complex with $Q$ at the place 0 and $P$ at the place 1 . Then we have for all $n \geq 0$ :

$$
N F \Gamma(P \rightarrow Q)_{n} \cong \mathrm{cr}_{n}(F)(P, \ldots, P) \oplus \mathrm{cr}_{n+1}(F)(Q, P, \ldots, P) ;
$$

the differential $\partial: N F \Gamma(P \rightarrow Q)_{n} \rightarrow N F \Gamma(P \rightarrow Q)_{n-1}$ is given by the following diagram:

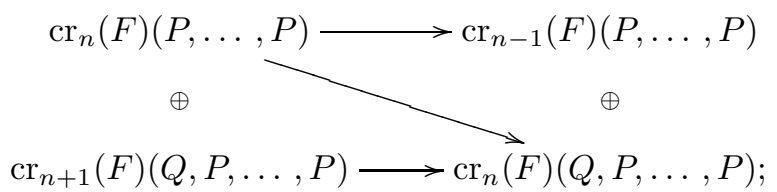

here, the upper horizontal map is the alternating sum $\sum_{i=1}^{n-1}(-1)^{i}\left(+_{\varepsilon^{i}}\right)$ of the plus maps associated with $\varepsilon^{1}=(2,1, \ldots, 1), \ldots, \varepsilon^{n-1}=(1, \ldots, 1,2)$; the lower horizontal map is $\left(\left(+{ }_{(2,1, \ldots, 1)}\right) \circ \mathrm{cr}_{n+1}(F)\left(\operatorname{id}_{Q}, f, \mathrm{id}_{P}, \ldots, \mathrm{id}_{P}\right)\right)+\sum_{i=1}^{n-1}(-1)^{i}\left(+{ }_{\left(1, \varepsilon^{i}\right)}\right)$; the diagonal map is $\operatorname{cr}_{n}(F)\left(f, \operatorname{id}_{P}, \ldots, \operatorname{id}_{p}\right)$.

Proof. By construction of $\Gamma$, we have $\Gamma(P \rightarrow Q)_{n} \cong Q \oplus P^{n}$ for all $n \geq 0$. By virtue of these isomorphisms, the maps $d_{i}: \Gamma(P \rightarrow Q)_{n} \rightarrow \Gamma(P \rightarrow Q)_{n-1}$ and $s_{i}: \Gamma(P \rightarrow Q)_{n} \rightarrow \Gamma(P \rightarrow Q)_{n+1}$ can be described as follows:

$$
\begin{aligned}
& d_{i}\left(q, p_{1}, \ldots, p_{n}\right)= \begin{cases}\left(q+f\left(p_{1}\right), p_{2}, \ldots, p_{n}\right) & \text { if } i=0 \\
\left(q, p_{1}, \ldots, p_{i-1}, p_{i}+p_{i+1}, p_{i+2}, \ldots, p_{n}\right) & \text { if } 1 \leq i \leq n-1 \\
\left(q, p_{1}, \ldots, p_{n-1}\right) & \text { if } i=n\end{cases} \\
& s_{i}\left(q, p_{1}, \ldots, p_{n}\right)=\left(q, p_{1}, \ldots, p_{i}, 0, p_{i+1}, \ldots, p_{n}\right) \quad \text { if } 0 \leq i \leq n
\end{aligned}
$$

(see also Lemma A.2 in $[\mathrm{JM}]$ ). Hence, by Proposition 1.2, we have

$$
\begin{aligned}
F \Gamma(P \rightarrow Q)_{n} \cong F(Q) \oplus F\left(P^{n}\right) \oplus \operatorname{cr}_{2}(F)\left(Q, P^{n}\right) \\
\cong \bigoplus_{k=0}^{n} \bigoplus\left(\begin{array}{c}
n \\
k
\end{array}\right) \\
\end{aligned}
$$

Thus we have

$$
\begin{aligned}
N F \Gamma(P \rightarrow Q)_{n} & \cong F \Gamma(P \rightarrow Q)_{n} /\left(\sum_{i=0}^{n-1} \operatorname{Im}\left(s_{i}\right)\right) \\
& \cong \operatorname{cr}_{n}(F)(P, \ldots, P) \oplus \operatorname{cr}_{n+1}(F)(Q, P, \ldots, P) .
\end{aligned}
$$

Finally, one can easily check now that the differential has the claimed form.

For any object $P$ in $\mathcal{P}$ and $k \geq 0$, we write $P[-k]$ for the complex which has $P$ at the place $k$ and 0 else. Lemma 2.2 states, in particular, that $N F \Gamma(P[0]) \cong F(P)[0]$ and that $N F \Gamma(P[-1])$ is isomorphic to the complex

$$
\ldots \longrightarrow \mathrm{cr}_{n}(F)(P, \ldots, P) \stackrel{\partial_{n}}{\longrightarrow} \mathrm{cr}_{n-1}(F)(P, \ldots, P) \stackrel{\partial_{n-1}}{\longrightarrow} \ldots \stackrel{\partial_{2}}{\longrightarrow} F(P) \longrightarrow 0
$$

where $\partial_{n}=\sum_{i=1}^{n-1}(-1)^{i}\left({ }_{\varepsilon^{i}}\right)$.

Now, we recall the definition of the Koszul complex (see I 4.3.1.3 in [I1], V.1.3 in [ABW], or section 2 in $[\mathrm{G}$ ]). Let $n \in \mathbb{N}$, let $R$ be a commutative ring, and let $f: P \rightarrow Q$ be a homomorphism between projective $R$-modules $P, Q$. 
Definition 2.3. For any $k \in\{0, \ldots, n+1\}$, let

$$
d_{k+1}: \Lambda^{k+1}(P) \otimes \operatorname{Sym}^{n-k-1}(Q) \rightarrow \Lambda^{k}(P) \otimes \operatorname{Sym}^{n-k}(Q)
$$

denote the Koszul differential given by

$$
\begin{aligned}
p_{1} & \wedge \ldots \wedge p_{k+1} \otimes q_{k+2} \cdots q_{n} \\
& \mapsto \sum_{i=1}^{k+1}(-1)^{k+1-i} p_{1} \wedge \ldots \wedge \hat{p}_{i} \wedge \ldots \wedge p_{k+1} \otimes f\left(p_{i}\right) q_{k+2} \cdots q_{n} .
\end{aligned}
$$

The complex

$$
0 \rightarrow \Lambda^{n}(P) \stackrel{d_{n}}{\rightarrow} \Lambda^{n-1}(P) \otimes Q \stackrel{d_{n-1}}{\rightarrow} \ldots \stackrel{d_{2}}{\rightarrow} P \otimes \operatorname{Sym}^{n-1}(Q) \stackrel{d_{1}}{\rightarrow} \operatorname{Sym}^{n}(Q) \rightarrow 0
$$

is called the $n$-th Koszul complex associated with $f$ and is denoted by $\operatorname{Kos}^{n}(f)$.

One can prove analogously to Lemme 2.1.2.1 on page 277 in $[12$ that the complexes $\operatorname{Kos}^{n}(f)$ and $N \operatorname{Sym}^{n} \Gamma(P \rightarrow Q)$ are isomorphic in the derived category of $R$. Whereas Illusie uses three complex homomorphisms (which cannot be composed since they have different directions) to realize this isomorphism; we now explicitly construct a single complex homomorphism $u^{n}(f)$ from $\operatorname{Kos}^{n}(f)$ to $N \operatorname{Sym}^{n} \Gamma(P \rightarrow Q)$ and show that $u^{n}(f)$ is a quasi-isomorphism. For $k=0, \ldots, n$, let $u_{k}^{n}(f)$ denote the composition

$$
\begin{aligned}
& \Lambda^{k}(P) \otimes \operatorname{Sym}^{n-k}(Q) \stackrel{a_{k} \otimes \mathrm{id}}{\longrightarrow} P^{\otimes k} \otimes \operatorname{Sym}^{n-k}(Q) \\
& \stackrel{(1.4)(a)}{\longrightarrow} \mathrm{cr}_{k}\left(\operatorname{Sym}^{k}\right)(P, \ldots, P) \otimes \operatorname{Sym}^{n-k}(Q) \\
& \stackrel{\operatorname{can}}{\longrightarrow} \bigoplus_{i=0}^{n-k} \mathrm{cr}_{k}\left(\operatorname{Sym}^{k+i}\right)(P, \ldots, P) \otimes \operatorname{Sym}^{n-k-i}(Q) \\
& \stackrel{(1.4)(a)}{\longrightarrow} \mathrm{cr}_{k}\left(\operatorname{Sym}^{n}\right)(P, \ldots, P) \oplus \mathrm{cr}_{k+1}\left(\operatorname{Sym}^{n}\right)(Q, P, \ldots, P) \\
& \stackrel{(2.2)}{\longrightarrow} N \operatorname{Sym}^{n} \Gamma(P \rightarrow Q)_{k} \text {; }
\end{aligned}
$$

here, $a_{k}: \Lambda^{k}(P) \rightarrow P^{\otimes k}, p_{1} \wedge \ldots \wedge p_{k} \mapsto \sum_{\sigma \in \Sigma_{k}}(-1)^{\operatorname{sgn}(\sigma)} p_{\sigma(1)} \otimes \ldots \otimes p_{\sigma(k)}$, denotes the antisymmetrization map; the other homomorphisms are explained under the indicated example or lemma.

Proposition 2.4. The homomorphisms $u_{k}^{n}(f), k=0, \ldots, n$, define a quasi-isomorphism

$$
u^{n}(f): \operatorname{Kos}^{n}(f) \stackrel{\sim}{\rightarrow} N \operatorname{Sym}^{n} \Gamma(P \rightarrow Q) .
$$


Proof. We write $\mathrm{S}$ for Sym, and we set $L .:=N \mathrm{~S}^{n} \Gamma(P \rightarrow Q)$. The following picture illustrates $u^{n}(f)$ :

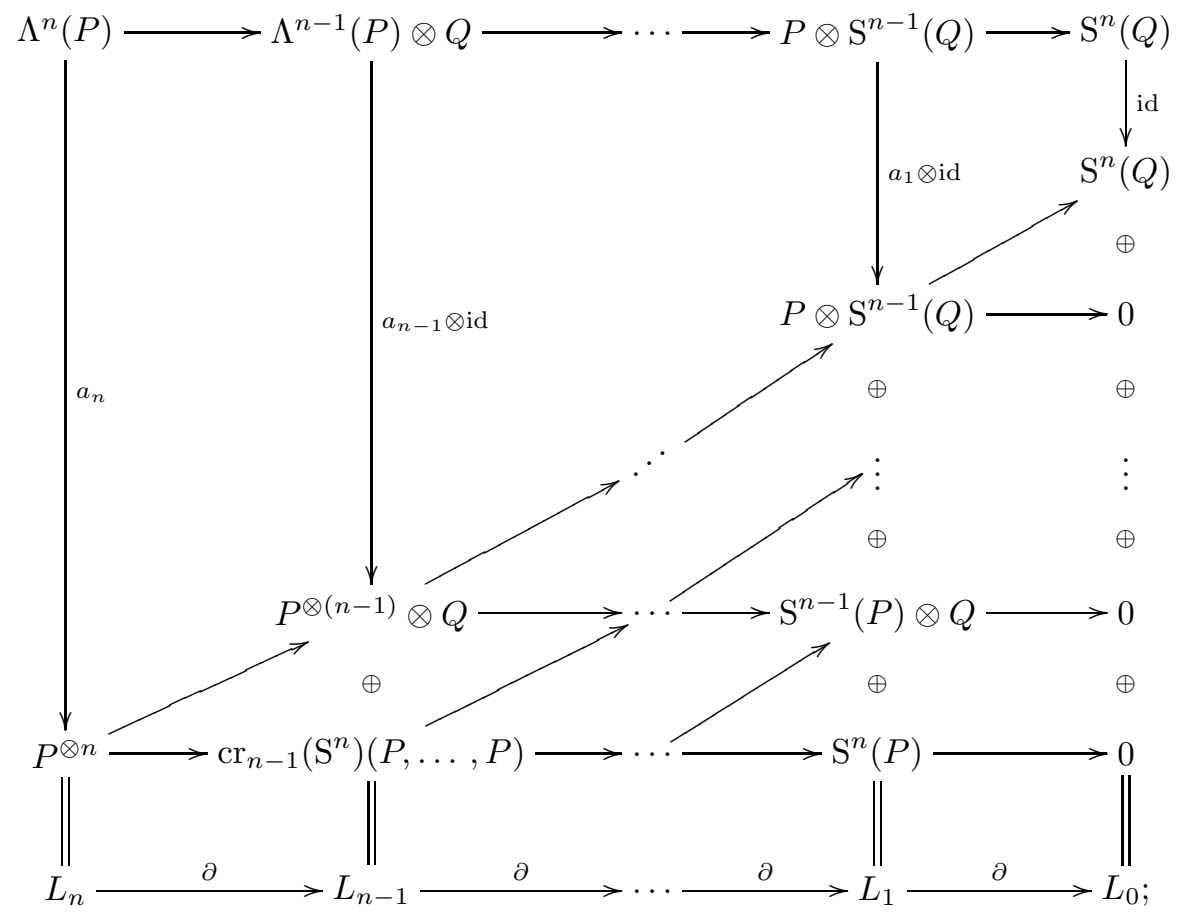

here, the differentials in the middle complex are given by Lemma 2.2. For all $k=0, \ldots, n$, the composition

$$
\begin{aligned}
\Lambda^{k}(P) \stackrel{a_{k}}{\longrightarrow} P^{\otimes k} & \cong \mathrm{cr}_{k}\left(\mathrm{~S}^{k}\right)(P, \ldots, P)^{\stackrel{\sum_{i=1}^{k-1}(-1)^{i}\left(+_{\varepsilon^{i}}\right)}{\longrightarrow} \operatorname{cr}_{k-1}\left(\mathrm{~S}^{k}\right)(P, \ldots, P)} \\
& \cong\left(\mathrm{S}^{2}(P) \otimes P \otimes \ldots \otimes P\right) \oplus \ldots \oplus\left(P \otimes \ldots \otimes P \otimes \mathrm{S}^{2}(P)\right)
\end{aligned}
$$

is obviously the zero map; furthermore, one easily sees that the diagram

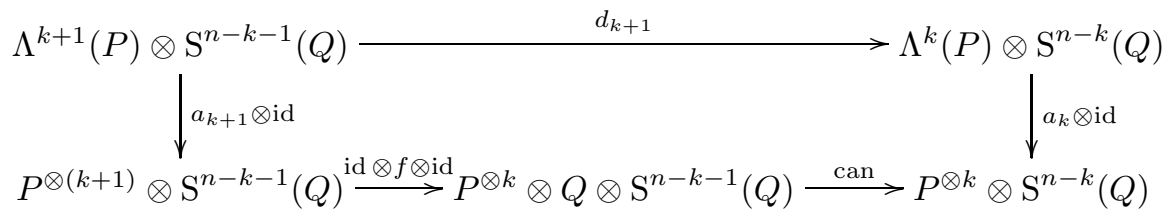

commutes. This proves that $u$ is a complex homomorphism. Obviously, the complexes $\operatorname{Kos}^{n}(f)$ and $N \mathrm{~S}^{n} \Gamma(P \rightarrow Q)$ can be filtered by subcomplexes such that $u^{n}(f)$ is compatible with these filtrations and such that, for $k=0, \ldots, n$, the map between the $k$-th successive quotients induced by $u^{n}(f)$ is the complex homomorphism

$$
u^{k}(0) \otimes \mathrm{id}: \Lambda^{k}(P)[-k] \otimes \mathrm{S}^{n-k}(Q) \rightarrow N \mathrm{~S}^{k} \Gamma(P \rightarrow 0) \otimes \mathrm{S}^{n-k}(Q) .
$$

Thus it suffices to show that, for all $n \geq 0$, the complex homomorphism

$$
u^{n}(0): \Lambda^{n}(P)[-n] \rightarrow N \mathrm{~S}^{n} \Gamma(P \rightarrow 0)
$$


is a quasi-isomorphism, i.e., that Proposition 2.4 is true in the case $Q=0$. We prove this by induction on $n$. For $n=0$ this is clear. So let $n \geq 1$. The homomorphism $u^{n}\left(\operatorname{id}_{P}\right)$ is a quasi-isomorphism since $\operatorname{Kos}^{n}\left(\operatorname{id}_{P}\right)$ is exact and the complex $N \mathrm{~S}^{n} \Gamma(P \stackrel{\text { id }}{\rightarrow} P)$ is homotopic to the zero complex. Furthermore, the complex homomorphisms

$$
u^{k}(0) \otimes \mathrm{id}: \Lambda^{k}(P)[-k] \otimes \mathrm{S}^{n-k}(P) \rightarrow N \mathrm{~S}^{k} \Gamma(P \rightarrow 0) \otimes \mathrm{S}^{n-k}(P)
$$

$k=0, \ldots, n-1$, are quasi-isomorphisms by the induction hypothesis. Using the filtrations already considered above, we obtain that $u^{n}(0)$ is a quasi-isomorphism. (Alternatively, this also follows from Proposition I 4.3.2.1(i) on p. 111 in [I 1] and the following fact which is a little bit tedious to prove: The complex homomorphism $N \mathrm{~S}^{n} \Gamma(P \rightarrow 0) \rightarrow \Lambda^{n}(P)[-n]$ constructed in [1] is left inverse to $u^{n}(0)$.) This ends the proof of Proposition 2.4.

Any commutative square

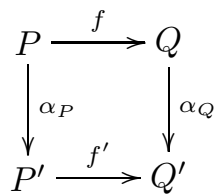

of homomorphisms between projective $R$-modules induces a homomorphism

$$
\operatorname{Kos}^{n}(\alpha): \operatorname{Kos}^{n}(f) \rightarrow \operatorname{Kos}^{n}\left(f^{\prime}\right)
$$

between the corresponding Koszul complexes in the obvious way.

Corollary 2.5. If $\left(\alpha_{P}^{1}, \alpha_{Q}^{1}\right)$ and $\left(\alpha_{P}^{2}, \alpha_{Q}^{2}\right)$ are homotopic complex homomorphisms

between the complexes $P \stackrel{f}{\rightarrow} Q$ and $P^{\prime} \stackrel{f^{\prime}}{\rightarrow} Q^{\prime}$, then the induced homomorphisms between the $k$-th homology modules of the corresponding $n$-th Koszul complexes are equal:

$$
H_{k}\left(\operatorname{Kos}^{n}\left(\alpha^{1}\right)\right)=H_{k}\left(\operatorname{Kos}^{n}\left(\alpha^{2}\right)\right): H_{k}\left(\operatorname{Kos}^{n}(f)\right) \rightarrow H_{k}\left(\operatorname{Kos}^{n}\left(f^{\prime}\right)\right)
$$

(for all $k \geq 0$ ).

Proof. Obviously, the diagram

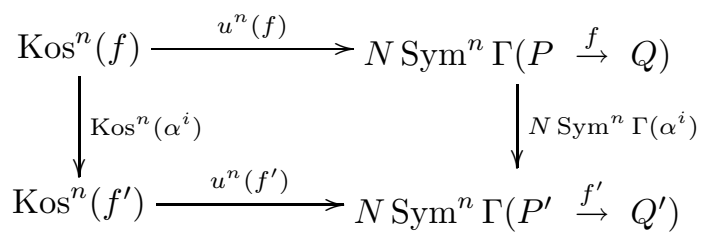

commutes for $i=1,2$. Furthermore, the complex homomorphisms $N \operatorname{Sym}^{n} \Gamma\left(\alpha^{1}\right)$ and $N$ Sym ${ }^{n} \Gamma\left(\alpha^{2}\right)$ are homotopic to each other. Thus, Corollary 2.5 follows from Proposition 2.4.

Remark 2.6. In the next section, we will use only Corollary 2.5 (and not Proposition 2.4). The simplicial methods used here to prove Corollary 2.5 are actually not necessary: Alternatively, one can use use Lemma 2.2 to define the complex $N F \Gamma(P \rightarrow Q)$ and one can directly show that homotopic complex homomorphisms between $P \rightarrow Q$ and $P^{\prime} \rightarrow Q^{\prime}$ induce homotopic complex homomorphisms between $N F \Gamma(P \rightarrow Q)$ and $N F \Gamma\left(P^{\prime} \rightarrow Q^{\prime}\right)$. However, the simplicial definition of $N F \Gamma(K$. 
has the advantage that it yields a more natural argument for the proof of Corollary 2.5 and that it can be applied also to complexes $K$. of length $>1$ (see also section $6)$.

Remark 2.7. One can prove analogously to Proposition 2.4 that the Koszul complex

$$
\tilde{\operatorname{Kos}}^{n}(f): 0 \rightarrow D^{n}(P) \rightarrow D^{n-1}(P) \otimes Q \rightarrow \ldots \rightarrow P \otimes \Lambda^{n-1}(Q) \rightarrow \Lambda^{n}(Q) \rightarrow 0
$$

(defined analogously to 2.3) is quasi-isomorphic to the complex $N \Lambda^{n} \Gamma(P \rightarrow Q$ ).

\section{Computation of the homology of Koszul complexes}

Let $R$ be a commutative ring and $n \in \mathbb{N}$. In this section, we compute the homology modules of the $n$-th Koszul complex $\operatorname{Kos}^{n}(f)$ for each homomorphism $f: P \rightarrow Q$ between $\mathrm{f}$. g. projective $R$-modules which has the following properties: $f$ is injective and $Q / f(P)$ is an f. g. projective $R / I$-module for some ideal $I$ in $R$ which is locally generated by a nonzero-divisor.

First, we recall the definition of the Schur modules corresponding to Young diagrams of hook type.

Definition 3.1. Let $k \in\{0, \ldots, n\}$ and $V$ an f. g. projective $R$-module. Let

$$
d_{k+1}: \Lambda^{k+1}(V) \otimes \operatorname{Sym}^{n-k-1}(V) \rightarrow \Lambda^{k}(V) \otimes \operatorname{Sym}^{n-k}(V)
$$

denote the differential in the Koszul complex $\operatorname{Kos}^{n}\left(\mathrm{id}_{V}\right)$ (see Definition 2.3). The module

$$
L_{k}^{n}(V):=\operatorname{Im}\left(d_{k+1}\right) \subseteq \Lambda^{k}(V) \otimes \operatorname{Sym}^{n-k}(V)
$$

is called a Schur module associated with $V$ and the Young diagram $(k+1,1, \ldots, 1)$ $((n-k-1) 1 \mathrm{~s})$.

Since $\operatorname{Kos}^{n}\left(\operatorname{id}_{V}\right)$ is exact, $L_{k}^{n}(V)$ is an f. g. projective $R$-module for all $k=$ $0, \ldots, n$. For instance, we have $L_{0}^{n}(V) \cong \operatorname{Sym}^{n}(V), L_{n-1}^{n}(V) \cong \Lambda^{n}(V)$, and $L_{n}^{n}(V) \cong 0$. Obviously, the definition given here agrees with the definition on p. 220 in $\mathrm{ABW}$.

Theorem 3.2. Let $I$ be an ideal in $R$ which is locally generated by a nonzerodivisor. Let $0 \rightarrow P \stackrel{f}{\rightarrow} Q \rightarrow V \rightarrow 0$ be an $R$-projective resolution of an $f$. g. projective $R / I$-module $V$. Then, for all $k=0, \ldots, n$, we have a canonical isomorphism

$$
H_{k}\left(\operatorname{Kos}^{n}(f)\right) \cong L_{k}^{n}(V) \otimes\left(I / I^{2}\right)^{\otimes k}
$$

between the $k$-th homology module of the $n$-th Koszul complex and the tensor product of the Schur module $L_{k}^{n}(V)$ with the $k$-th tensor power of the conormal module $I / I^{2}$. In particular, $H_{k}\left(\operatorname{Kos}^{n}(f)\right)$ is a projective $R / I$-module.

Remark 3.3. We consider the following extreme cases:

(a) If $k=n$, Theorem 3.2 states that the differential

$$
d_{n}: \Lambda^{n}(P) \rightarrow \Lambda^{n-1}(P) \otimes Q
$$

is injective. This immediately follows from the exactness of the Koszul complex $\operatorname{Kos}^{n}\left(\operatorname{id}_{P}\right)$ and the injectivity of $f$.

(b) If $k=0$, Theorem 3.2 states that the sequence

$$
P \otimes \operatorname{Sym}^{n-1}(Q) \stackrel{d_{1}}{\longrightarrow} \operatorname{Sym}^{n}(Q) \stackrel{\text { can }}{\longrightarrow} \operatorname{Sym}^{n}(V) \longrightarrow 0
$$


is exact. This already follows from the construction of symmetric powers. In both cases, the assumption on the ideal $I$ is not used.

Proof of Theorem 3.2. We fix $k \in\{0, \ldots, n\}$. For any f. g. projective $R / I$-module $V$, we choose an $R$-projective resolution $0 \rightarrow P_{V} \stackrel{f_{V}}{\rightarrow} Q_{V} \rightarrow V \rightarrow 0$ of $V$. For any homomorphism $\alpha: V \rightarrow W$ between $\mathrm{f}$. g. projective $R / I$-modules $V, W$, there is a complex homomorphism

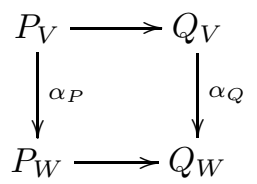

which is compatible with $\alpha$; this complex homomorphism is unique up to homotopy by a standard argument of homological algebra. Thus, by Corollary 2.5 , we obtain a well-defined functor

$$
F: \mathcal{P}_{R / I} \rightarrow(R \text {-modules }), \quad V \mapsto H_{k}\left(\operatorname{Kos}^{n}\left(f_{V}\right)\right) .
$$

Let $G$ denote the functor

$$
G: \mathcal{P}_{R / I} \rightarrow(R \text {-modules }), \quad V \mapsto L_{k}^{n}(V) \otimes\left(I / I^{2}\right)^{\otimes k} .
$$

We have to show that $F(V) \cong G(V)$ for all $V \in \mathcal{P}_{R / I}$. For this, we verify the assumptions of Theorem 1.5.

One easily checks (see also 4.3.1.5 on p. 109 in [1]) that, for all $f: P \rightarrow Q$, $f^{\prime}: P^{\prime} \rightarrow Q^{\prime}$ in $\mathcal{P}_{R}$, we have

$$
\operatorname{Kos}^{n}\left(f \oplus f^{\prime}\right) \cong \bigoplus_{n_{1}+n_{2}=n} \operatorname{Kos}^{n_{1}}(f) \otimes \operatorname{Kos}^{n_{2}}\left(f^{\prime}\right) .
$$

(The tensor product of two complexes $K$. and $L$. is a priori only a double complex. In this paper, $K . \otimes L$. denotes both the double complex and the total complex of this double complex.) Using Proposition 1.2, we obtain the following isomorphism for all $i \geq 0$ and $V_{1}, \ldots, V_{i} \in \mathcal{P}_{R / I}$ (see also Example 1.4(a)):

$$
\operatorname{cr}_{i}(F)\left(V_{1}, \ldots, V_{i}\right) \cong \bigoplus_{\substack{\left(n_{1}, \ldots, n_{i}\right) \in\{1, \ldots, n\}^{i} \\ n_{1}+\ldots+n_{i}=n}} H_{k}\left(\operatorname{Kos}^{n_{1}}\left(f_{V_{1}}\right) \otimes \ldots \otimes \operatorname{Kos}^{n_{i}}\left(f_{V_{i}}\right)\right) .
$$

In particular, $F$ is of degree $\leq n$ and, for $V_{1}=\ldots=V_{i}=R / I$, we obtain

$$
\operatorname{cr}_{i}(F)(R / I, \ldots, R / I) \cong \bigoplus_{\substack{\left(n_{1}, \ldots, n_{i}\right) \in\{1, \ldots, n\}^{i} \\ n_{1}+\ldots+n_{i}=n}} H_{k}\left((I \stackrel{\text { can }}{\longrightarrow} R)^{\otimes i}\right)
$$

since $\operatorname{Kos}^{m}(I \rightarrow R) \cong(I \rightarrow R)$ for all $m \geq 1$. The complex $(I \stackrel{\text { can }}{\longrightarrow} R)^{\otimes i}$ has the shape

$$
0 \rightarrow I^{\otimes i} \rightarrow \bigoplus^{\left(\begin{array}{c}
i \\
i-1
\end{array}\right)} I^{\otimes(i-1)} \rightarrow \ldots \rightarrow \bigoplus^{\left(\begin{array}{c}
i \\
1
\end{array}\right)} I \rightarrow R \rightarrow 0 ;
$$

here, the differential $\bigoplus^{\left(\begin{array}{c}i \\ k\end{array}\right)} I^{\otimes k} \rightarrow \bigoplus^{\left(\begin{array}{c}i \\ k-1\end{array}\right)} I^{\otimes(k-1)}$ is the composition of the differential $\bigoplus^{\left(\begin{array}{c}i \\ k\end{array}\right)} I^{\otimes k} \rightarrow \bigoplus^{\left(\begin{array}{c}i \\ k-1\end{array}\right)} I^{\otimes k}$ in the complex $(R \stackrel{\text { id }}{\rightarrow} R)^{\otimes i} \otimes I^{\otimes k}$ with the canonical inclusion $\bigoplus^{\left(\begin{array}{c}i \\ k-1\end{array}\right)} I^{\otimes k} \rightarrow \bigoplus^{\left(\begin{array}{c}i \\ k-1\end{array}\right)} I^{\otimes(k-1)}$. Hence, the module $Z_{k}\left((I \rightarrow R)^{\otimes i}\right)$ of $k$-cycles is isomorphic to $Z_{k}\left((R \rightarrow R)^{\otimes i}\right) \otimes I^{\otimes k}$, and the module $B_{k}\left((I \rightarrow R)^{\otimes i}\right)$ 
of $k$-boundaries is isomorphic to $B_{k}\left((R \rightarrow R)^{\otimes i}\right) \otimes I^{\otimes(k+1)}$. Since $(R \rightarrow R)^{\otimes i}$ is exact, we obtain

$$
H_{k}\left((I \rightarrow R)^{\otimes i}\right) \cong B_{k}\left((R \rightarrow R)^{\otimes i}\right) \otimes\left(I / I^{2}\right)^{\otimes k} .
$$

Similarly, for the functor $G$, we obtain

$$
\stackrel{\operatorname{cr}_{i}(G)\left(V_{1}, \ldots, V_{i}\right)}{\cong} \bigoplus_{\substack{\left(n_{1}, \ldots, n_{i}\right)\left\{\{1, \ldots, n\}^{i} \\ n_{1}+\ldots+n_{i}=n\right.}} B_{k}\left(\operatorname{Kos}^{n_{1}}\left(\operatorname{id}_{V_{1}}\right) \otimes \ldots \otimes \operatorname{Kos}^{n_{i}}\left(\operatorname{id}_{V_{i}}\right)\right) \otimes\left(I / I^{2}\right)^{\otimes k}
$$

In particular, $G$ is of degree $\leq n$ and we have for $V_{1}=\ldots=V_{i}=R / I$ :

$$
\operatorname{cr}_{i}(G)(R / I, \ldots, R / I) \cong \bigoplus_{\substack{\left(n_{1}, \ldots, n_{i}\right) \in\{1, \ldots, n\}^{i} \\ n_{1}+\ldots+n_{i}=n}} B_{k}\left((R / I \stackrel{\text { id }}{\rightarrow} R / I)^{\otimes i}\right) \otimes\left(I / I^{2}\right)^{\otimes k}
$$

Thus, for all $i \geq 1$, we obtain an isomorphism

$$
\alpha_{i}(R / I, \ldots, R / I): \mathrm{cr}_{i}(F)(R / I, \ldots, R / I) \stackrel{\sim}{\rightarrow} \operatorname{cr}_{i}(G)(R / I, \ldots, R / I) .
$$

The multiplication with $\bar{r} \in R / I$ in the $l$-th component of $\operatorname{cr}_{i}(F)(R / I, \ldots, R / I)$ obviously corresponds to the endomorphism

$$
\bigoplus_{\left(n_{1}, \ldots, n_{i}\right)} r^{n_{i}} \quad \text { of } \bigoplus_{\left(n_{1}, \ldots, n_{i}\right)} B_{k}\left((R \rightarrow R)^{\otimes i}\right) \otimes\left(I / I^{2}\right)^{\otimes k}
$$

The analogous statement also holds for $G$. Thus, the maps $\alpha_{i}(R / I, \ldots, R / I)$, $i=1, \ldots, n$, are compatible with the action of $R / I$ in each component. For any $\varepsilon \in\{1, \ldots, n\}^{i}$ and $V_{1}, \ldots, V_{i} \in \mathcal{P}_{R / I}$, the diagonal map

$$
\Delta_{\varepsilon}: \operatorname{cr}_{i}(F)\left(V_{1}, \ldots, V_{i}\right) \rightarrow \mathrm{cr}_{|\varepsilon|}(F)\left(V_{1}, \ldots, V_{1}, \ldots, V_{i}, \ldots, V_{i}\right)
$$

is induced by an obvious complex homomorphism

$$
\begin{aligned}
\Delta_{\varepsilon}: \bigoplus_{\left(n_{1}, \ldots, n_{i}\right)} \operatorname{Kos}^{n_{1}}\left(f_{V_{1}}\right) & \otimes \ldots \otimes \operatorname{Kos}^{n_{i}}\left(f_{V_{i}}\right) \\
& \rightarrow \bigoplus_{\left(n_{1}, \ldots, n_{|\varepsilon|}\right)} \operatorname{Kos}^{n_{1}}\left(f_{V_{1}}\right) \otimes \ldots \otimes \operatorname{Kos}^{n_{|\varepsilon|}}\left(f_{V_{i}}\right) .
\end{aligned}
$$

Similarly, the diagonal map

$$
\Delta_{\varepsilon}: \operatorname{cr}_{i}(G)\left(V_{1}, \ldots, V_{i}\right) \rightarrow \mathrm{cr}_{|\varepsilon|}(G)\left(V_{1}, \ldots, V_{1}, \ldots, V_{i}, \ldots, V_{i}\right)
$$

is induced by an obvious complex homomorphism

$$
\begin{aligned}
\Delta_{\varepsilon}: \bigoplus_{\left(n_{1}, \ldots, n_{i}\right)} \operatorname{Kos}^{n_{1}}\left(\operatorname{id}_{V_{1}}\right) & \otimes \ldots \otimes \operatorname{Kos}^{n_{i}}\left(\operatorname{id}_{V_{i}}\right) \\
& \rightarrow \bigoplus_{\left(n_{1}, \ldots, n_{|\varepsilon|}\right)} \operatorname{Kos}^{n_{1}}\left(\operatorname{id}_{V_{1}}\right) \otimes \ldots \otimes \operatorname{Kos}^{n_{|\varepsilon|}}\left(\operatorname{id}_{V_{i}}\right) .
\end{aligned}
$$

Using these facts, one easily sees that the isomorphisms $\alpha_{i}(R / I, \ldots, R / I), i \geq 1$, are compatible with the diagonal maps. In the same way one can prove that these isomorphisms are compatible with the plus maps. Hence, by Theorem 1.5, the functors $F$ and $G$ are isomorphic, and Theorem 3.2 is proved.

In the next proposition, we explicitly describe the isomorphism of Theorem 3.2. 
Proposition 3.4. The isomorphism

$$
L_{k}^{n}(V) \otimes I^{k} / I^{k+1} \stackrel{\sim}{\rightarrow} H_{k}\left(\operatorname{Kos}^{n}(f)\right)
$$

constructed in the proof of Theorem 3.2 maps the element

$$
d_{k+1}\left(v_{1} \wedge \ldots \wedge v_{k+1} \otimes v_{k+2} \cdots v_{n}\right) \otimes \overline{r_{1} \cdots r_{k}}
$$

to the homology class of the element

$$
\sum_{i=1}^{k+1}(-1)^{k+1-i}\left(r_{1} q_{1}\right) \wedge \ldots \wedge \hat{q}_{i} \wedge \ldots \wedge\left(r_{k} q_{k+1}\right) \otimes q_{i} q_{k+2} \cdots q_{n} ;
$$

here, $q_{i}$ is a preimage of $v_{i}$ under the given surjective map $Q \rightarrow V($ for $i=1, \ldots, n)$ and, for any $r \in I$ and $q \in Q$, the element $r q$ of $Q$ is considered as an element of $P$.

Proof. The isomorphism $G \stackrel{\sim}{\rightarrow} F$ constructed in the proof of Theorem 3.2 induces an isomorphism $\mathrm{cr}_{n}(G) \stackrel{\sim}{\rightarrow} \mathrm{cr}_{n}(F)$ of $n$-functors. Obviously, the diagrams

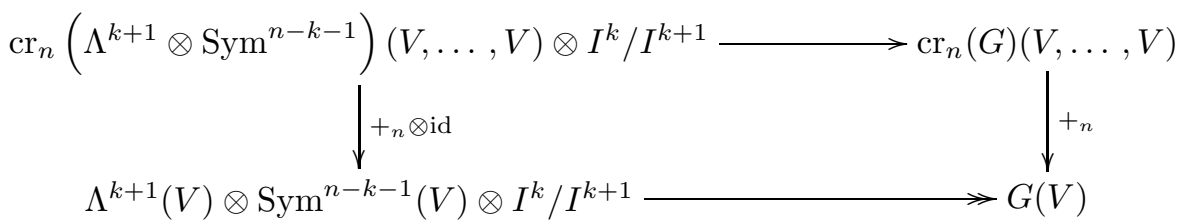

(where both horizontal maps are induced by $d_{k+1}$ ) and

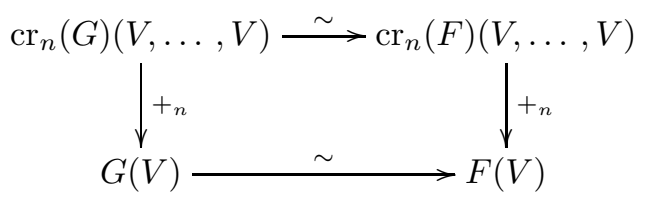

commute for all $V \in \mathcal{P}_{R / I}$. The $R / I$-module $\operatorname{cr}_{n}\left(\Lambda^{k+1} \otimes \operatorname{Sym}^{n-k-1}\right)(V, \ldots, V)$ is isomorphic to a direct sum of $\left(\begin{array}{c}n \\ k+1\end{array}\right)$ copies of $V^{\otimes n}$. We index these summands by the subsets $T$ of $\{1, \ldots, n\}$ with $k+1$ elements in the canonical way. For $v_{1} \otimes \ldots \otimes v_{n} \in V^{\otimes n}$ and $T=\left\{t_{1}<\ldots<t_{k+1}\right\}$, let $\left(v_{1} \otimes \ldots \otimes v_{n}\right)[T]$ denote the corresponding element of $\operatorname{cr}_{n}\left(\Lambda^{k+1} \otimes \operatorname{Sym}^{n-k-1}\right)(V, \ldots, V)$. We obviously have

$$
\begin{aligned}
& +_{n}\left(\left(v_{1} \otimes \ldots \otimes v_{n}\right)[T]\right) \\
& \quad=v_{t_{1}} \wedge \ldots \wedge v_{t_{k+1}} \otimes \prod_{t \in\{1, \ldots, n\} \backslash T} v_{t} \quad \text { in } \quad \Lambda^{k+1}(V) \otimes \operatorname{Sym}^{n-k-1}(V) .
\end{aligned}
$$

In particular, the restriction of ${ }_{n}{ }_{n}$ to the direct summand which corresponds e.g. to $\{1, \ldots, k+1\}$ is surjective. Thus it suffices to show that the composition of the upper horizontal maps of the above diagrams with the right vertical map in the lower diagram maps the element $\left(v_{1} \otimes \ldots \otimes v_{n}\right)[\{1, \ldots, k+1\}] \otimes \overline{r_{1} \cdots r_{k}}$ to the homology class of the element given in Proposition 3.4. We obviously have

$$
\begin{aligned}
\operatorname{cr}_{n}\left(d_{k+1}\right)(V, \ldots, V)\left(\left(v_{1} \otimes \ldots \otimes v_{n}\right)[\{1, \ldots, k+1\}]\right) \\
\quad=\sum_{i=1}^{k+1}(-1)^{k+1-i}\left(v_{1} \otimes \ldots \otimes v_{n}\right)[\{1, \ldots, \hat{i}, \ldots, k+1\}]
\end{aligned}
$$


in $\mathrm{cr}_{n}\left(\Lambda^{k} \otimes \operatorname{Sym}^{n-k}\right)(V, \ldots, V)$. The isomorphism

$$
\mathrm{cr}_{n}(G)(V, \ldots, V) \stackrel{\sim}{\rightarrow} \mathrm{cr}_{n}(F)(V, \ldots, V)
$$

is equal to the composition

$$
\begin{aligned}
& \operatorname{cr}_{n}(G)(V, \ldots, V) \cong V^{\otimes n} \otimes \operatorname{cr}_{n}(G)(R / I, \ldots, R / I) \\
& \quad \cong V^{\otimes n} \otimes \operatorname{cr}_{n}(F)(R / I, \ldots, R / I) \cong \mathrm{cr}_{n}(F)(V, \ldots, V)
\end{aligned}
$$

where the middle isomorphism has been explicitly constructed in the proof of Theorem 3.2 and the exterior isomorphisms have been introduced in Example 1.7. The composition

$$
\begin{aligned}
& \operatorname{Im}\left(\operatorname{cr}_{n}\left(d_{k+1}\right)(R / I, \ldots, R / I)\right) \otimes I^{k} / I^{k+1} \\
& \quad \cong \operatorname{cr}_{n}(G)(R / I, \ldots, R / I) \cong \operatorname{cr}_{n}(F)(R / I, \ldots, R / I)
\end{aligned}
$$

maps the element $\mathrm{cr}_{n}\left(d_{k+1}\right)(R / I, \ldots, R / I)(1[\{1, \ldots, k+1\}]) \otimes \overline{r_{1} \cdots r_{k}}$ to the homology class of the element

$$
\sum_{i=1}^{k+1}(-1)^{k+1-i}\left(r_{1} \otimes \ldots \otimes r_{i-1} \otimes 1 \otimes r_{i} \otimes \ldots \otimes r_{k} \otimes 1 \otimes \ldots \otimes 1\right)[\{1, \ldots, \hat{i}, \ldots, k+1\}]
$$

in the complex $(I \rightarrow R)^{\otimes n}$ (see the proof of Theorem 3.2). Furthermore, the isomorphism $V^{\otimes n} \otimes \mathrm{cr}_{n}(F)(R / I, \ldots, R / I) \cong \mathrm{cr}_{n}(F)(V, \ldots, V)$ maps the element $v_{1} \otimes \ldots \otimes v_{n} \otimes x$ to the image of $x$ under the map

$$
\operatorname{cr}_{n}(F)\left(v_{1}, \ldots, v_{n}\right): \mathrm{cr}_{n}(F)(R / I, \ldots, R / I) \rightarrow \mathrm{cr}_{n}(F)(V, \ldots, V) .
$$

(This easily follows from Example 1.7). Hence, the composition of the upper horizontal maps in the above diagrams maps the element

$$
\left(v_{1} \otimes \ldots \otimes v_{n}\right)[\{1, \ldots, k+1\}] \otimes \overline{r_{1} \cdots r_{k}}
$$

to the homology class of the element

$$
\begin{aligned}
& \sum_{i=1}^{k+1}(-1)^{k+1-i}\left(\left(r_{1} q_{1}\right) \otimes \ldots \otimes\left(r_{i-1} q_{i-1}\right) \otimes q_{i}\right. \\
& \left.\quad \otimes\left(r_{i} q_{i+1}\right) \otimes \ldots \otimes\left(r_{k} q_{k+1}\right) \otimes q_{k+2} \otimes \ldots \otimes q_{n}\right)[\{1, \ldots, \hat{i}, \ldots, k+1\}]
\end{aligned}
$$

in the complex $(P \rightarrow Q)^{\otimes n}$. The plus map $+_{n}: \operatorname{cr}_{n}(F)(V, \ldots, V) \rightarrow F(V)$ maps the latter homology class to the homology class stated in Proposition 3.4. Thus, Proposition 3.4 is proved.

In the following remark, we sketch an alternative proof of Theorem 3.2.

Remark 3.5. An easy, but tedious calculation shows directly that the association described in Proposition 3.4 yields a well-defined homomorphism

$$
G(V)=L_{k}^{n}(V) \otimes I^{k} / I^{k+1} \rightarrow H_{k}\left(\operatorname{Kos}^{n}(f)\right)=F(V)
$$

for all $V \in \mathcal{P}_{R / I}$. Furthermore, one can verify that the induced homomorphisms $\operatorname{cr}_{i}(G)(R / I, \ldots, R / I) \rightarrow \mathrm{cr}_{i}(F)(R / I, \ldots, R / I), i \geq 1$, are bijective. By Proposition 1.2, this implies that the morphism $G \rightarrow F$ of functors is an isomorphism. So, in this proof, the abstract argument Theorem 1.5 has been replaced by more explicit computations which, from the combinatorial point of view, are more complicated. 
Remark 3.6. Analogously to Theorem 3.2, one can prove the following statement for the Koszul complex $\tilde{\operatorname{Kos}}^{n}(f)$ introduced in Remark 2.7: Under the assumptions of Theorem 3.2, we have

$$
H_{k}\left(\tilde{\operatorname{Kos}}^{n}(f)\right) \cong \tilde{L}_{k}^{n}(V) \otimes\left(I / I^{2}\right)^{\otimes k} \quad \text { for all } k \geq 0 ;
$$

here, $\tilde{L}_{k}^{n}(V)$ denotes the co-Schur module of type $(k+1,1, \ldots, 1)$ which can be defined analogously to 3.1 (see also Definition II 1.3 on p. 220 in [ABW]).

\section{A new proof of the Adams-Riemann-Roch formula FOR REGULAR CLOSED IMMERSIONS}

In this section, we deduce the Adams-Riemann-Roch theorem for regular closed immersions of codimension 1 from the main result of the previous section. Using blowing up and the excess intersection formula, the Adams-Riemann-Roch theorem for regular closed immersions of arbitrary codimension then follows from this as in SGA 6. In particular, we obtain an easy, elementary and natural proof of the Adams-Riemann-Roch theorem which does not use the deformation to the normal cone any longer.

First, we recall the following definitions and facts of the theory of $\lambda$-rings (see Expose V in [SGA 6], section 4.5 in [S], or Chapter I in [FL]). Let $K$ be a (special) $\lambda$-ring, $C \in K$ of (finite) $\lambda$-degree $d$, and $x \in K$ arbitrary. Furthermore, let $\mu$ be a polynomial in the $\lambda$-operations $\lambda_{1}, \lambda_{2}, \ldots$ without constant term. Then, there is a unique element $\mu(C, x) \in K$ which is functorial in $(K, C, x)$ and which has the following property:

$$
\mu\left(x \cdot \lambda_{-1}(C)\right)=\mu(C, x) \cdot \lambda_{-1}(C)
$$

(here, $\lambda_{-1}(C):=\sum_{i=0}^{d}(-1)^{i} \lambda_{i}(C)$ ). This immediately follows from the following fact which is easy to prove (cf. Lemma 5.2 in Expose $\mathrm{V}$ of [SGA 6]): If the elements $\lambda_{1}(C), \ldots, \lambda_{d}(C)$ and $\lambda_{1}(x), \lambda_{2}(x), \ldots$ are considered as indeterminates and if the polynomial ring $\mathbb{Z}\left[\lambda_{1}(C), \ldots, \lambda_{d}(C), \lambda_{1}(x), \lambda_{2}(x), \ldots\right]$ is equipped with the obvious $\lambda$-structure, then the element $\mu\left(x \cdot \lambda_{-1}(C)\right)$ is divisible by $\lambda_{-1}(C)$. For $n \geq 1$, let $\psi_{n}$ denote the $n$-th Adams operation, i.e., $\psi_{n}=N_{n}\left(\lambda_{1}, \ldots, \lambda_{n}\right)$ where $N_{n}$ denotes the $n$-th Newton polynomial. Furthermore, let $\theta^{n}(C)$ denote the $n$-th Bott element of $C$ (see $\S 4$ in [K1] or p. 24 in [FL]). The $n$-th symmetric power operation is inductively defined by $\sigma_{0} \equiv 1$ and $\sigma_{n}=\sum_{i=1}^{n}(-1)^{i-1} \lambda_{i} \sigma_{n-i}$ for $n \geq 1$. For $0 \leq k \leq n$, the Schur operation $s_{k}^{n}$ of type $(k+1,1, \ldots, 1)$ is defined by $s_{k}^{n}=\sum_{i=k+1}^{n}(-1)^{i-k-1} \lambda_{i} \sigma_{n-i}$.

Lemma 4.1. (a) Let $\mu$ be the product of two polynomials $\mu_{1}$ and $\mu_{2}$. Then we have

$$
\mu(C, x)=\mu_{1}(C, x) \cdot \mu_{2}(C, x) \cdot \lambda_{-1}(C) .
$$

(b) For all $n \geq 0$ and $x, y \in K$, we have

$$
\sigma_{n}(C, x+y)=\sigma_{n}(C, x)+\sum_{i=1}^{n-1} \sigma_{i}(C, x) \cdot \sigma_{n-i}(C, y) \cdot \lambda_{-1}(C)+\sigma_{n}(C, y) .
$$

(c) For all $n \geq 1$ we have $\psi_{n}(C, x)=\theta^{n}(C) \cdot \psi_{n}(x)$.

(d) Let $d=1$. Then we have for all $n \geq 1$,

$$
\sigma_{n}(C, x)=\sum_{k=0}^{n-1}(-1)^{k} s_{k}^{n}(x) C^{k} .
$$

In particular, we have $\sigma_{n}(1, x)=\psi_{n}(x)$. 
Proof. The assertions (a) and (b) immediately follow from the definition. The assertion (c) follows from the multiplicativity of $\psi_{n}$. If $d=1$, then we have

$$
\begin{aligned}
\sigma_{n}(x & \left.\cdot \lambda_{-1}(C)\right)=\sigma_{n}(x \cdot(1-C))=\sum_{i=0}^{n} \sigma_{n-i}(x) \cdot \sigma_{i}(-x C) \\
& =\sum_{i=0}^{n}(-1)^{i} \sigma_{n-i}(x) \cdot \lambda_{i}(x C) \quad\left(\text { since } \sigma_{i}(-x)=(-1)^{i} \lambda_{i}(x)\right) \\
& =\sum_{i=0}^{n}(-1)^{i} \sigma_{n-i}(x) \cdot \lambda_{i}(x) \cdot C^{i} \quad(\text { since } C \text { is of } \lambda \text {-degree } 1) \\
& =\sum_{i=0}^{n}(-1)^{i} \sigma_{n-i}(x) \cdot \lambda_{i}(x) \cdot\left(C^{i}-1\right) \quad\left(\text { since } \sum_{i=0}^{n}(-1)^{i} \sigma_{n-i}(x) \cdot \lambda_{i}(x)=0\right) \\
& =\sum_{i=0}^{n}(-1)^{i+1} \sigma_{n-i}(x) \cdot \lambda_{i}(x) \cdot\left(1+C+\ldots+C^{i-1}\right) \cdot \lambda_{-1}(C) \quad \text { (geom. sum). }
\end{aligned}
$$

Hence we have

$$
\begin{aligned}
\sigma_{n}(C, x)=\sum_{i=1}^{n}(-1)^{i+1} \sigma_{n-i}(x) \cdot \lambda_{i}(x) \cdot\left(1+C+\ldots+C^{i-1}\right) \\
=\sum_{k=0}^{n-1}\left(\sum_{i=k+1}^{n}(-1)^{i+1} \sigma_{n-i}(x) \cdot \lambda_{i}(x)\right) C^{k}=\sum_{k=0}^{n-1}(-1)^{k} s_{k}^{n}(x) C^{k} .
\end{aligned}
$$

In particular, we have $\sigma_{n}(1, x)=\sum_{k=0}^{n-1}(-1)^{k} s_{k}^{n}(x)=\psi_{n}(x)$ by section 3 in [G].

Now, let $X$ be a noetherian scheme with the property that each coherent $\mathcal{O}_{X^{-}}$ module is a quotient of a locally free $\mathcal{O}_{X}$-module (of finite type). Let $K_{0}(X)$ denote the Grothendieck group of all locally free $\mathcal{O}_{X}$-modules and $K_{0}^{\infty}(X)$ the Grothendieck group of all coherent $\mathcal{O}_{X}$-modules which have a finite resolution by locally free $\mathcal{O}_{X}$-modules. By Proposition 4.1 on p. 126 in [FL], the canonical homomorphism $K_{0}(X) \rightarrow K_{0}^{\infty}(X)$ is bijective. Let $i: Y \hookrightarrow X$ be a regular closed immersion of codimension $d$ with conormal sheaf $\mathcal{C}$. Then, for any locally free $\mathcal{O}_{Y^{-}}$ module $\mathcal{V}$, the direct image $i_{*}(\mathcal{V})$ is a coherent $\mathcal{O}_{X}$-module which has a finite locally free resolution (see p. 127 in $[\mathrm{FL})$. Thus, the association $[\mathcal{V}] \mapsto\left[i_{*}(\mathcal{V})\right]$ induces a well-defined homomorphism

$$
i_{*}: K_{0}(Y) \rightarrow K_{0}^{\infty}(X) \cong K_{0}(X) .
$$

The following formula describes the behavior of products with respect to this homomorphism. It follows from the self-intersection formula and it will be used in our proof of the Adams-Riemann-Roch formula.

Proposition 4.2. For all $x, y \in K_{0}(Y)$, we have

$$
i_{*}(x) \cdot i_{*}(y)=i_{*}\left(x \cdot y \cdot \lambda_{-1}([\mathcal{C}])\right) \quad \text { in } \quad K_{0}(X) .
$$

Proof. See Corollaire 2.8 on p. 436 in [SGA 6].

The assertion (a) of the following theorem has already been proved in Théorème 4.3 on p. 449 in [SGA 6], however, only modulo torsion. The claimed version without denominators has been proved in Théorème 2.1 on p. 24 in [J] using the deformation to the normal cone. The Adams-Riemann-Roch formula given in (b) follows also 
from Théorème 2.1 on p. 24 in [J] and it has been proved in Theorem 6.3 on p. 142 in $\mathrm{FL}$.

Theorem 4.3. Let $n \geq 1$.

(a) (Riemann-Roch formula for $\sigma_{n}$ ) For all $y \in K_{0}(Y)$ we have

$$
\sigma_{n}\left(i_{*}(y)\right)=i_{*}\left(\sigma_{n}([\mathcal{C}], y)\right) \quad \text { in } \quad K_{0}(X) .
$$

(b) (Adams-Riemann-Roch formula) For all $y \in K_{0}(Y)$ we have

$$
\psi_{n}\left(i_{*}(y)\right)=i_{*}\left(\theta^{n}([\mathcal{C}]) \cdot \psi_{n}(y)\right) \quad \text { in } \quad K_{0}(X) .
$$

Proof. (a) First, let $d=1$. By Lemma 4.1(b) and Proposition 4.2, the elements $y$ of $K_{0}(Y)$ for which the Riemann-Roch formula for $\sigma_{n}$ holds for all $n \geq 1$ is a subgroup of $K_{0}(Y)$. Thus, it suffices to prove the Riemann-Roch formula for $y=[\mathcal{V}]$ where $\mathcal{V}$ is a locally free $\mathcal{O}_{Y}$-module. We choose a locally free resolution $0 \rightarrow \mathcal{P} \stackrel{f}{\rightarrow} \mathcal{Q} \rightarrow i_{*}(\mathcal{V}) \rightarrow 0$ of $i_{*}(\mathcal{V})$ on $X$. By gluing the isomorphisms of Theorem 3.2 , we obtain isomorphisms

$$
\mathcal{H}_{k}\left(\operatorname{Kos}^{n}(f)\right) \cong i_{*}\left(L_{k}^{n}(\mathcal{V}) \otimes \mathcal{C}^{\otimes k}\right), \quad k=0, \ldots, n .
$$

Hence, we have in $K_{0}^{\infty}(X)$,

$$
\sum_{k=0}^{n}(-1)^{k}\left[\Lambda^{k}(\mathcal{P}) \otimes \operatorname{Sym}^{n-k}(\mathcal{Q})\right]=i_{*}\left(\sum_{k=0}^{n-1}(-1)^{k}\left[L_{k}^{n}(\mathcal{V}) \otimes \mathcal{C}^{\otimes k}\right]\right) .
$$

Thus, we obtain

$$
\begin{aligned}
& \sigma_{n}\left(i_{*}([\mathcal{V}])\right)=\sigma_{n}([\mathcal{Q}]-[\mathcal{P}])=\sum_{k=0}^{k}(-1)^{k}\left[\Lambda^{k}(\mathcal{P}) \otimes \operatorname{Sym}^{n-k}(\mathcal{Q})\right] \\
& \quad=i_{*}\left(\sum_{k=0}^{n-1}(-1)^{k} s_{k}^{n}([\mathcal{V}]) \cdot[\mathcal{C}]^{k}\right)=i_{*}\left(\sigma_{n}([\mathcal{C}],[\mathcal{V}])\right) \text { in } \quad K_{0}(X) ;
\end{aligned}
$$

here, we have used the exactness of the Koszul complex $\operatorname{Kos}^{n}\left(\mathrm{id}_{\mathcal{V}}\right)$ and Lemma 4.1(d). This proves assertion (a) in the case $d=1$. The general case can be deduced from this as on p. 449 and p. 450 in [SGA 6] using blowing up and the excess intersection formula.

(b) Let $\psi_{n}=\sum_{\nu} a_{\nu} \sigma_{1}^{\nu_{1}} \cdots \sigma_{n}^{\nu_{n}}$ be the representation of $\psi_{n}$ as a polynomial in the symmetric power operations $\sigma_{1}, \ldots, \sigma_{n}$. Then we have in $K_{0}(X)$,

$$
\begin{aligned}
& \psi_{n}\left(i_{*}(y)\right)=\sum_{\nu} a_{\nu} \sigma_{1}\left(i_{*}(y)\right)^{\nu_{1}} \cdots \sigma_{n}\left(i_{*}(y)\right)^{\nu_{n}} \\
& =\sum_{\nu} a_{\nu} i_{*}\left(\sigma_{1}([\mathcal{C}], y)\right)^{\nu_{1}} \cdots i_{*}\left(\sigma_{n}([\mathcal{C}], y)\right)^{\nu_{n}} \quad(\text { Theorem 4.3(a)) } \\
& =i_{*}\left(\sum_{\nu} a_{\nu} \sigma_{1}([\mathcal{C}], y)^{\nu_{1}} \cdots \sigma_{n}([\mathcal{C}], y)^{\nu_{n}} \cdot \lambda_{-1}([\mathcal{C}])^{\nu_{1}+\ldots+\nu_{n}-1}\right) \\
& =i_{*}\left(\psi_{n}([\mathcal{C}], y)\right) \quad(\text { Lemma } 4.1(\mathrm{a})) \\
& =i_{*}\left(\theta^{n}([\mathcal{C}]) \cdot \psi_{n}(y)\right) \quad(\text { Lemma } 4.1(\mathrm{c})) .
\end{aligned}
$$

This ends the proof of Theorem 4.3. 


\section{RiEMANN-Roch FOR TENSOR POWERS}

In the paper [K2], we have established Riemann-Roch formulas for tensor power operations. In the case of regular closed immersions, we have used the deformation to the normal cone to prove them. The aim of this section is to prove these formulas by using the method developed in the previous sections. In contrast to the previous sections, this method can be applied here to regular closed immersions not only of codimension 1 but of arbitrary codimension.

Let $R$ be a noetherian ring and $n \in \mathbb{N}$. Let $\Sigma_{n}$ denote the $n$-th symmetric group, i.e., the group of permutations of $I_{n}:=\{1, \ldots, n\}$. We write $R\left[I_{n}\right]$ for the direct sum of $n$ copies of $R$ together with the obvious action of $\Sigma_{n}$. The $\Sigma_{n}$-module $K_{n}$ is defined by the short exact sequence

$$
0 \longrightarrow K_{n} \longrightarrow R\left[I_{n}\right] \stackrel{\text { sum }}{\longrightarrow} R \longrightarrow 0 .
$$

Let $I$ be an ideal in $R$ which is locally generated by a regular sequence of length $d$. Furthermore, let $P$. $\rightarrow V$ be an $R$-projective resolution of an $\mathrm{f}$. g. projective $R / I$-module $V$. We view the total complex $P{ }^{\otimes n}$ of the $n$-th tensor power of the complex $P$. as a complex of $\Sigma_{n}$-modules in the canonical way. In particular, the homology modules $H_{k}\left(P{ }^{\otimes n}\right), k \geq 0$, are equipped with a canonical $\Sigma_{n}$-action.

Theorem 5.1. For all $k \geq 0$, there is a canonical $\Sigma_{n}$-isomorphism

$$
H_{k}\left(P .{ }^{\otimes n}\right) \cong V^{\otimes n} \otimes \Lambda^{k}\left(I / I^{2} \otimes K_{n}\right) ;
$$

here, $V^{\otimes n}$ is considered as a $\Sigma_{n}$-module with the natural action and $I / I^{2}$ as a $\Sigma_{n}$ module with the trivial action. In particular, $H_{k}\left(P{ }^{\otimes n}\right)$ is a projective $R / I$-module.

Proof. We fix $k \in\{0, \ldots, n\}$. A homotopy between two complex homomorphisms $f, g: P \longrightarrow Q$. induces a (non-equivariant) homotopy between the complex homomorphisms $f^{\otimes n}, g^{\otimes n}: P .{ }^{\otimes n} \Longrightarrow Q .{ }^{\otimes n}$. In particular, the $\Sigma_{n}$-homomorphisms $H_{k}\left(f^{\otimes n}\right)$ and $H_{k}\left(g^{\otimes n}\right)$ coincide. Therefore, we obtain a well-defined functor

$$
F: \mathcal{P}_{R / I} \rightarrow\left(R\left[\Sigma_{n}\right] \text {-modules }\right), \quad V \mapsto H_{k}\left(P .{ }^{\otimes n}\right),
$$

as in the proof of Theorem 3.2. Let $G$ denote the functor

$$
G: \mathcal{P}_{R / I} \rightarrow\left(R\left[\Sigma_{n}\right] \text {-modules }\right), \quad V \mapsto V^{\otimes n} \otimes \Lambda^{k}\left(I / I^{2} \otimes K_{n}\right) .
$$

We have to show that $F(V) \cong G(V)$ for all $V \in \mathcal{P}_{R / I}$. Again, we verify the assumptions of Theorem 1.5.

First, we suppose that the ideal $I$ is globally generated by a regular sequence. This means that there is a homomorphism $\varepsilon: F \rightarrow R$ from a free $R$-module $F$ of rank $d$ to $R$ such that the Koszul complex $\operatorname{Kos}^{d}(\varepsilon)$ is a resolution of $R / I$ (by virtue of the canonical map $\left.\operatorname{Kos}^{d}(\varepsilon)_{0} \cong R \longrightarrow R / I\right)$. One easily checks that the complex $\left(\operatorname{Kos}^{d}(\varepsilon)\right)^{\otimes n}$ is $\Sigma_{n}$-isomorphic to the Koszul complex $\operatorname{Kos}^{n d}\left(F \otimes R\left[I_{n}\right] \stackrel{\operatorname{sum}}{\longrightarrow} F \stackrel{\varepsilon}{\longrightarrow}\right.$ $R)$. By Lemma 3.3 in [K2], the homology module $H_{k}\left(\operatorname{Kos}^{n d}\left(F \otimes R\left[I_{n}\right] \rightarrow F \rightarrow R\right)\right)$ is $\Sigma_{n}$-isomorphic to the $k$-th exterior power of

$$
\operatorname{ker}\left(F / I F \otimes R\left[I_{n}\right] \stackrel{\operatorname{sum}}{\longrightarrow} F / I F \stackrel{\bar{\varepsilon}}{\longrightarrow} I / I^{2}\right) \cong F / I F \otimes K_{n} \cong I / I^{2} \otimes K_{n} .
$$

Thus, we have established a $\Sigma_{n}$-isomorphism $F(R / I) \cong \Lambda^{k}\left(I / I^{2} \otimes K_{n}\right)$. It obviously does not depend on the chosen homomorphism $\varepsilon$. Hence, we can glue these isomorphisms and obtain a canonical $\Sigma_{n}$-isomorphism $F(R / I) \cong \Lambda^{k}\left(I / I^{2} \otimes K_{n}\right)$ 
also in the case the ideal $I$ is only locally generated by a regular sequence. For any two complexes $P$. and $Q$., we have

$$
(P . \oplus Q .)^{\otimes n} \cong \bigoplus_{n_{1}+n_{2}=n} \operatorname{Ind}_{\Sigma_{n_{1}} \times \Sigma_{n_{2}}}^{\Sigma_{n}}\left(P .{ }^{\otimes n_{1}} \otimes Q .{ }^{\otimes n_{2}}\right) .
$$

Hence, as in the proof of Theorem 3.2, we obtain for all $i \geq 0$,

$$
\begin{aligned}
& \operatorname{cr}_{i}(F)(R / I, \ldots, R / I) \cong \bigoplus_{\substack{\left(n_{1}, \ldots, n_{i}\right) \in\{1, \ldots, n\}^{i} \\
n_{1}+\ldots+n_{i}=n}} \operatorname{Ind}_{\Sigma_{n_{1}} \times \ldots \times \Sigma_{n_{i}}}^{\Sigma_{n}} \operatorname{Res}_{\Sigma_{n_{1}} \times \ldots \times \Sigma_{n_{i}}}^{\Sigma_{n}} F(R / I) \\
& \cong\left(\bigoplus_{\left(n_{1}, \ldots, n_{i}\right)} \operatorname{Ind}_{\Sigma_{n_{1}} \times \ldots \times \Sigma_{n_{i}}}^{\Sigma_{n}}(R)\right) \otimes \Lambda^{k}\left(I / I^{2} \otimes K_{n}\right) \\
& \cong \operatorname{cr}_{i}(G)(R / I, \ldots, R / I) \text {. }
\end{aligned}
$$

One easily sees that these $\Sigma_{n}$-isomorphisms are compatible with the action of $R / I$ in each component and with the diagonal and plus maps. Thus, Theorem 5.1 follows from Theorem 1.5.

Example 5.2. Let $n=2$. Then Theorem 5.1 yields the well-known $\Sigma_{2}$-isomorphism

$$
\operatorname{Tor}_{k}^{R}(V, V) \cong V^{\otimes 2} \otimes \Lambda^{k}\left(\left(I / I^{2}\right)_{\mathrm{sgn}}\right)
$$

where the transposition in $\Sigma_{2}$ acts by -1 on $\left(I / I^{2}\right)_{\operatorname{sgn}}$.

Now, let $X$ be a noetherian scheme with the property that each coherent $\mathcal{O}_{X^{-}}$ module is a quotient of a locally free $\mathcal{O}_{X}$-module. Let $K_{0}\left(\Sigma_{n}, X\right)$ denote the Grothendieck group of all locally free $\Sigma_{n}$-modules on $X$. Using the "binomial theorem"

$$
(\mathcal{F} \oplus \mathcal{G})^{\otimes n} \cong \bigoplus_{n_{1}+n_{2}=n} \operatorname{Ind}_{\Sigma_{n_{1}} \times \Sigma_{n_{2}}}^{\Sigma_{n}}\left(\mathcal{F}^{\otimes n_{1}} \otimes \mathcal{G}^{\otimes n_{2}}\right)
$$

the association $[\mathcal{F}] \mapsto\left[\mathcal{F}^{\otimes n}\right]$ can be canonically extended to a map

$$
\tau_{n}: K_{0}(X) \rightarrow K_{0}\left(\Sigma_{n}, X\right)
$$

(see section $1 \mathrm{in} \mathrm{K} 2$ ). It is called the $n$-th tensor power operation. Let $i: Y \hookrightarrow X$ be a regular closed immersion of codimension $d$ with conormal sheaf $\mathcal{C}$. Let $i_{*}$ denote both the homomorphism from $K_{0}(Y)$ to $K_{0}(X)$ introduced in section 4 and the homomorphism from $K_{0}\left(\Sigma_{n}, Y\right)$ to $K_{0}\left(\Sigma_{n}, X\right)$ defined analogously. The following theorem is Theorem 4.1 of [K2].

Theorem 5.3 (Riemann-Roch formula for $\left.\tau_{n}\right)$. For all $y \in K_{0}(Y)$, we have

$$
\tau_{n}\left(i_{*}(y)\right)=i_{*}\left(\lambda_{-1}\left(\left[\mathcal{C} \otimes K_{n}\right]\right) \cdot \tau_{n}(y)\right) \quad \text { in } \quad K_{0}\left(\Sigma_{n}, X\right) .
$$


Proof. If Theorem 5.3 is true for $y_{1}, y_{2} \in K_{0}(Y)$ and for all $n \geq 1$, then it is also true for $y_{1}+y_{2}$. This follows from the following calculation $\left(\Sigma_{n_{1}, n_{2}}:=\Sigma_{n_{1}} \times \Sigma_{n_{2}}\right)$ :

$$
\begin{aligned}
\tau_{n}\left(i_{*}\left(y_{1}+y_{2}\right)\right)=\sum_{n_{1}+n_{2}=n} \operatorname{Ind}_{\Sigma_{n_{1}, n_{2}}}^{\Sigma_{n}}\left(\tau_{n_{1}}\left(i_{*}\left(y_{1}\right)\right) \cdot \tau_{n_{2}}\left(i_{*}\left(y_{2}\right)\right)\right) \\
\quad=\sum \operatorname{Ind}_{\Sigma_{n_{1}, n_{2}}^{\Sigma_{n}}}^{\Sigma_{n}}\left(i_{*}\left(\lambda_{-1}\left(\left[\mathcal{C} \otimes K_{n_{1}}\right]\right) \cdot \tau_{n_{1}}\left(y_{1}\right)\right) \cdot i_{*}\left(\lambda_{-1}\left(\left[\mathcal{C} \otimes K_{n_{2}}\right]\right) \cdot \tau_{n_{2}}\left(y_{2}\right)\right)\right) \\
=\sum \operatorname{Ind}_{\Sigma_{n_{1}, n_{2}}}^{\Sigma_{n}}\left(i_{*}\left(\lambda_{-1}\left(\left[\mathcal{C} \otimes K_{n_{1}}\right]\right) \lambda_{-1}\left(\left[\mathcal{C} \otimes K_{n_{2}}\right]\right) \lambda_{-1}([\mathcal{C}]) \tau_{n_{1}}\left(y_{1}\right) \tau_{n_{2}}\left(y_{2}\right)\right)\right) \\
=\sum \operatorname{Ind}_{\Sigma_{n_{1}, n_{2}}}^{\Sigma_{n}}\left(i_{*}\left(\operatorname{Res}_{\Sigma_{n_{1}, n_{2}}}^{\Sigma_{n}} \lambda_{-1}\left(\left[\mathcal{C} \otimes K_{n}\right]\right) \cdot \tau_{n_{1}}\left(y_{1}\right) \cdot \tau_{n_{2}}\left(y_{2}\right)\right)\right) \\
=i_{*}\left(\lambda_{-1}\left(\left[\mathcal{C} \otimes K_{n}\right]\right) \cdot \sum_{n_{1}+n_{2}=n} \operatorname{Ind}_{\Sigma_{n_{1}, n_{2}}}^{\Sigma_{n}} \tau_{n_{1}}\left(y_{1}\right) \cdot \tau_{n_{2}}\left(y_{2}\right)\right) \\
=i_{*}\left(\lambda_{-1}\left(\left[\mathcal{C} \otimes K_{n}\right]\right) \cdot \tau_{n}\left(y_{1}+y_{2}\right)\right) .
\end{aligned}
$$

(Here, we have used Proposition 4.2 and Frobenius reciprocity.) Thus, it suffices to prove Theorem 5.3 for $y=[\mathcal{V}]$ where $\mathcal{V}$ is a locally free $\mathcal{O}_{Y}$-module. Let $\mathcal{P}$. $\rightarrow i_{*}(\mathcal{V})$ be a locally free resolution of $i_{*}(\mathcal{V})$ on $X$. By gluing the isomorphisms constructed in Theorem 5.1, we obtain $\Sigma_{n}$-isomorphisms

$$
\mathcal{H}_{k}\left(\mathcal{P} .{ }^{\otimes n}\right) \cong i_{*}\left(\mathcal{V}^{\otimes n} \otimes \Lambda^{k}\left(\mathcal{C} \otimes K_{n}\right)\right), \quad k \geq 0 .
$$

Hence, we have in $K_{0}\left(\Sigma_{n}, X\right)$ :

$$
\begin{aligned}
& \tau_{n}\left(i_{*}([\mathcal{V}])\right)=\tau_{n}\left(\sum_{i \geq 0}(-1)^{k}\left[\mathcal{P}_{k}\right]\right)=\sum_{k \geq 0}(-1)^{k}\left[\left(\mathcal{P} \cdot{ }^{\otimes n}\right)_{k}\right] \\
& \quad=\sum_{k \geq 0}(-1)^{k} i_{*}\left(\left[\mathcal{V}^{\otimes n} \otimes \Lambda^{k}\left(\mathcal{C} \otimes K_{n}\right)\right]\right)=i_{*}\left(\lambda_{-1}\left(\left[\mathcal{C} \otimes K_{n}\right]\right) \cdot \tau_{n}([\mathcal{V}])\right) .
\end{aligned}
$$

This proves Theorem 5.3.

\section{Some Computations for Regular Immersions of Higher Codimension}

Let $X$ be a noetherian scheme with the property that each coherent $\mathcal{O}_{X}$-module is a quotient of a locally free $\mathcal{O}_{X}$-module. Let $i: Y \hookrightarrow X$ be a regular closed immersion of codimension $d$ with conormal sheaf $\mathcal{C}$ and $n \in \mathbb{N}$. In this section, we study the question whether, in the case $d \geq 2$, the Riemann-Roch formula for $\sigma_{n}$ and $i_{*}$ (cf. Theorem 4.3(a)) can be proved directly with the methods developed in the previous sections (i.e., without using blowing up). More precisely, for any locally free $\mathcal{O}_{Y}$-module $\mathcal{V}$ and any locally free resolution $\mathcal{P} . \rightarrow i_{*}(\mathcal{V})$ of $i_{*}(\mathcal{V})$ on $X$, we are looking for a complex $\mathcal{K} .^{n}$ of locally free $\mathcal{O}_{X}$-modules such that we have

$$
\sigma_{n}\left(i_{*}([\mathcal{V}])\right) \stackrel{\text { def }}{=} \sigma_{n}\left(\sum_{k \geq 0}(-1)^{k}\left[\mathcal{P}_{k}\right]\right)=\sum_{k \geq 0}(-1)^{k}\left[\mathcal{K}_{k}^{n}\right] \quad \text { in } \quad K_{0}(X)
$$

and

$$
\sigma_{n}([\mathcal{C}],[\mathcal{V}])=\sum_{k \geq 0}(-1)^{k}\left[\mathcal{H}_{k}\left(\mathcal{K} .^{n}\right)\right] \quad \text { in } \quad K_{0}(Y)
$$

(or at least in the relative $K_{0}$-group of $Y$ in $X$, see [TT]). The existence of such a complex would immediately imply the Riemann-Roch formula for $\sigma_{n}$ (see the proof 
of Theorem 4.3(a)). In the case $d=1$, we have used the $\operatorname{complex} \operatorname{Kos}^{n}\left(\mathcal{P}_{1} \rightarrow \mathcal{P}_{0}\right)$ (see section 4) which is quasi-isomorphic to the complex $N \operatorname{Sym}^{n} \Gamma\left(\mathcal{P}_{1} \rightarrow \mathcal{P}_{0}\right)$ by Proposition 2.4. In section 5, we have used the complex $\mathcal{P} .{ }^{\otimes n}$ which is quasiisomorphic to the complex $N\left(\Gamma(\mathcal{P} .)^{\otimes n}\right.$ ) by the Eilenberg-Zilber theorem (see Application 8.5.3 on p. 277 in $[\overline{\mathrm{W}}]$ ). Thus, a natural candidate for the above complex $\mathcal{K} .{ }^{n}$ is the complex $\mathcal{K} .{ }^{n}:=N \operatorname{Sym}^{n} \Gamma(\mathcal{P}$.). We prove in Lemma 6.1 that this complex satisfies at least the first condition $\sigma_{n}\left(i_{*}([\mathcal{V}])\right)=\sum_{k>0}(-1)^{k}\left[\mathcal{K}_{k}^{n}\right]$. In Lemma 6.2 , we give a representation of $\sigma_{2}([\mathcal{C}],[\mathcal{V}])$ as an alternating sum which suggests the following conjecture: $\mathcal{H}_{k}\left(\mathcal{K}^{2}\right) \cong \operatorname{Sym}^{2}(\mathcal{V}) \otimes \Lambda^{k}(\mathcal{C})$ for $k$ even and $\mathcal{H}_{k}\left(\mathcal{K}^{2}{ }^{2}\right) \cong \Lambda^{2}(\mathcal{V}) \otimes \Lambda^{k}(\mathcal{C})$ for $k$ odd. In fact, this conjecture is true if 2 is invertible on $X$ (see Corollary 6.3). Theorem 6.4 states that, in the case $d=2$, we have $\mathcal{H}_{0}\left(\mathcal{K}^{2}{ }^{2}\right) \cong \operatorname{Sym}^{2}(\mathcal{V}), \mathcal{H}_{1}\left(\mathcal{K} .^{2}\right) \cong \Lambda^{2}(\mathcal{V}) \otimes \mathcal{C}$, however $\mathcal{H}_{2}\left(\mathcal{K} .^{2}\right) \cong D^{2}(\mathcal{V}) \otimes \Lambda^{2}(\mathcal{V})$, i.e., the conjecture is false for $k=2$. Nevertheless, this theorem implies the second condition for the complex $\mathcal{K}^{2}$ in the case $d=2$ since $\left[D^{2}(\mathcal{V})\right]=\left[\operatorname{Sym}^{2}(\mathcal{V})\right]$ in $K_{0}(Y)$. In the case $d>2, \mathcal{H}_{k}\left(\mathcal{K} .^{2}\right)$ probably has non-trivial 2-torsion for $k \geq 2$ (see Remark 6.5). In particular, it is not clear how to compute $\mathcal{H}_{k}\left(\mathcal{K}^{2}{ }^{2}\right)$ in general and to check the second condition for the complex $\mathcal{K}^{2}$. On the other hand, it might be possible to compute $\mathcal{H}_{k}\left(\mathcal{K} .^{n}\right)$ in the case $d=2$ even for all $n \geq 2$ and to verify the second condition for $\mathcal{K}^{n}$ in this case (see Example 6.6). I hope to say more on this in a future paper.

For any finite complex $\mathcal{P}$. of locally free $\mathcal{O}_{X}$-modules let $[\mathcal{P}$.] denote the Euler characteristic $\sum_{i \geq 0}(-1)^{i}\left[\mathcal{P}_{i}\right] \in K_{0}(X)$.

Lemma 6.1. Let $\mathcal{P}$. be a finite complex of locally free $\mathcal{O}_{X}$-modules. Then we have for all $n \geq 0$,

$$
\left[N \operatorname{Sym}^{n} \Gamma(\mathcal{P} .)\right]=\sigma_{n}([\mathcal{P} .]) \quad \text { in } \quad K_{0}(X) .
$$

Proof. One easily checks that the complex $N \operatorname{Sym}^{n} \Gamma(\mathcal{P}$.) has finite length. Furthermore, the $\mathcal{O}_{X}$-modules $N \operatorname{Sym}^{n} \Gamma(\mathcal{P} .)_{k}, k \geq 0$, are locally free (see section 2 in $[\mathrm{JM}]$ ). Therefore, the expression $\left[N \operatorname{Sym}^{n} \Gamma(\mathcal{P}).\right] \in K_{0}(X)$ is well-defined. First, we suppose that $\mathcal{P}$. is the complex $\mathcal{P}[-l]$ where $\mathcal{P}$ is a locally free $\mathcal{O}_{X}$-module and $l \in \mathbb{N}_{0}$. We have to show that $\sigma_{n}\left((-1)^{l}[\mathcal{P}]\right)=\left[N \operatorname{Sym}^{n} \Gamma(\mathcal{P}[-l])\right]$ in $K_{0}(X)$. We prove this by induction on $l$. For $l=0,1$ this follows for example from Proposition 2.4. We write $C(\mathcal{P}[-l])$ for the mapping cone of the identity of the complex $\mathcal{P}[-l]$. The obvious short exact sequence

$$
0 \rightarrow \mathcal{P}[-l] \rightarrow C(\mathcal{P}[-l]) \rightarrow \mathcal{P}[-l-1] \rightarrow 0
$$

of complexes induces a natural filtration of the complex $N \operatorname{Sym}^{n} \Gamma(C(\mathcal{P}[-l]))$ whose successive quotients are the complexes $N\left(\operatorname{Sym}^{i} \Gamma(\mathcal{P}[-l]) \otimes \operatorname{Sym}^{n-i} \Gamma(\mathcal{P}[-l-1])\right)$, $i=0, \ldots, n$. The complex $N \operatorname{Sym}^{n} \Gamma(C(\mathcal{P}[-l]))$ is homotopic to the zero complex (cf. section 2). For all $i=0, \ldots, n$, the complex

$$
N\left(\operatorname{Sym}^{i} \Gamma(\mathcal{P}[-l]) \otimes \operatorname{Sym}^{n-i} \Gamma(\mathcal{P}[-l-1])\right)
$$

is quasi-isomorphic to the complex $N \operatorname{Sym}^{i} \Gamma(\mathcal{P}[-l]) \otimes N \operatorname{Sym}^{n-i} \Gamma(\mathcal{P}[-l-1])$ by the Eilenberg-Zilber theorem (see Application 8.5.3 on p. 277 in [W]). Hence, we 
have for all $n \geq 1$,

$$
\begin{aligned}
\sum_{i=0}^{n}[ & \left.N \operatorname{Sym}^{i} \Gamma(\mathcal{P}[-l])\right] \cdot\left[N \operatorname{Sym}^{n-i} \Gamma(\mathcal{P}[-l-1])\right] \\
& =\sum_{i=0}^{n}\left[N \operatorname{Sym}^{i} \Gamma(\mathcal{P}[-l]) \otimes N \operatorname{Sym}^{n-i} \Gamma(\mathcal{P}[-l-1])\right] \\
& =\sum_{i=0}^{n}\left[N\left(\operatorname{Sym}^{i} \Gamma(\mathcal{P}[-l]) \otimes \operatorname{Sym}^{n-i} \Gamma(\mathcal{P}[-l-1])\right)\right] \\
& =N \operatorname{Sym}^{n} \Gamma(C(\mathcal{P}[-l]))=0 ;
\end{aligned}
$$

i.e., the power series $\sum_{i>0}\left[N \operatorname{Sym}^{i} \Gamma(\mathcal{P}[-l-1])\right] t^{i}$ is the inverse of the power series $\sum_{i \geq 0}\left[N \operatorname{Sym}^{i} \Gamma(\mathcal{P}[-l])\right] t^{i}$. Using the induction hypothesis, we obtain

$$
\left[N \operatorname{Sym}^{n} \Gamma(\mathcal{P}[-l-1])\right]=\sigma_{n}(-[\mathcal{P}[-l]])=\sigma_{n}\left((-1)^{l+1}[\mathcal{P}]\right) .
$$

(Alternatively, this can be deduced from Proposition 4.3.2.1(i) on p. 111 in [11.) Now, let $\mathcal{P}$. be an arbitrary complex of length $l$. We prove Lemma 6.1 by induction on $l$. Let $\sigma_{\leq l-1}(\mathcal{P}$.) denote the brutal truncation of $\mathcal{P}$.. The obvious short exact sequence

$$
0 \rightarrow \sigma_{\leq l-1}(\mathcal{P} .) \rightarrow \mathcal{P} . \rightarrow \mathcal{P}_{l}[-l] \rightarrow 0
$$

of complexes yields the equality

$$
\left[N \operatorname{Sym}^{n} \Gamma(\mathcal{P} .)\right]=\sum_{i=0}^{n}\left[N \operatorname{Sym}^{i} \Gamma\left(\sigma_{\leq l-1}(\mathcal{P} .)\right)\right] \cdot\left[N \operatorname{Sym}^{n-i} \Gamma\left(\mathcal{P}_{l}[-l]\right)\right] \quad \text { in } \quad K_{0}(X)
$$

as above. Using the induction hypothesis and the formula already proved, we finally obtain

$$
\left[N \operatorname{Sym}^{n} \Gamma(\mathcal{P} .)\right]=\sum_{i=0}^{n} \sigma_{i}\left(\sum_{i=0}^{l-1}(-1)^{i}\left[\mathcal{P}_{i}\right]\right) \cdot \sigma_{n-i}\left((-1)^{l}\left[\mathcal{P}_{l}\right]\right)=\sigma_{n}([\mathcal{P} .]) .
$$

Lemma 6.2. Let $K$ be a $\lambda$-ring, $C \in K$ of finite $\lambda$-degree $d$ and $x \in K$ arbitrary. Then we have

$$
\sigma_{2}(C, x)=\sigma_{2}(x) \lambda_{0}(C)-\lambda_{2}(x) \lambda_{1}(C)+\sigma_{2}(x) \lambda_{2}(C)-\lambda_{2}(x) \lambda_{3}(C)+\ldots \quad \text { in } \quad K .
$$

Proof. We prove Lemma 6.2 by induction on $d$. The case $d=1$ is a special case of Lemma 4.1(d). So, we may assume that $d \geq 2$ and that Lemma 6.2 holds for $d-1$. By the splitting principle (see Theorem 6.1 on p. 266 in [AT]), we may assume that $C=C^{\prime}+L$ where $C^{\prime} \in K$ is of $\lambda$-degree $d-1$ and $L \in K$ is of $\lambda$-degree 1 . Using the induction hypothesis, we obtain

$$
\begin{aligned}
& \sigma_{2}\left(\lambda_{-1}(C) x\right)=\sigma_{2}\left(\lambda_{-1}\left(C^{\prime}\right)(1-L) x\right) \\
& \quad=\left(\sigma_{2}((1-L) x)-\lambda_{2}((1-L) x) C^{\prime}+\sigma_{2}((1-L) x) \lambda_{2}\left(C^{\prime}\right)-\ldots\right) \lambda_{-1}\left(C^{\prime}\right) .
\end{aligned}
$$

Since

$$
\sigma_{2}((1-L) x)=\left(\sigma_{2}(x)-\lambda_{2}(x) L\right)(1-L)
$$

and

$$
\lambda_{2}((1-L) x)=\left(\lambda_{2}(x)-\sigma_{2}(x) L\right)(1-L),
$$


this implies

$$
\begin{aligned}
\sigma_{2}(C, x)= & \sigma_{2}(x)-\lambda_{2}(x) L-\lambda_{2}(x) C^{\prime}+\sigma_{2}(x) L C^{\prime} \\
& +\sigma_{2}(x) \lambda_{2}\left(C^{\prime}\right)-\lambda_{2}(x) L \lambda_{2}\left(C^{\prime}\right)-\ldots \\
= & \sigma_{2}(x)-\lambda_{2}(x) C+\sigma_{2}(x) \lambda_{2}(C)-\lambda_{2}(x) \lambda_{3}(C)+\ldots .
\end{aligned}
$$

Now, let $R$ be a commutative ring and $I$ an ideal in $R$ which is locally generated by a regular sequence of length $d$. For any $\mathrm{f}$. g. projective $R / I$-module $V$, we choose an $R$-projective resolution $P .(V)$ of $V$. The following statement is a consequence of Theorem 5.1.

Corollary 6.3. Let 2 be invertible in $R$. Then we have

$$
H_{k} N \operatorname{Sym}^{2} \Gamma(P .(V)) \cong \begin{cases}\operatorname{Sym}^{2}(V) \otimes \Lambda^{k}\left(I / I^{2}\right) & \text { for } k \text { even } \\ \Lambda^{2}(V) \otimes \Lambda^{k}\left(I / I^{2}\right) & \text { for } k \text { odd }\end{cases}
$$

Proof. By the Eilenberg-Zilber theorem, we have

$$
H_{k} N \operatorname{Sym}^{2} \Gamma(P .(V)) \cong H_{k} N\left(\Gamma(P .(V))^{\otimes 2}\right)^{\Sigma_{2}} \cong H_{k}\left(P .(V)^{\otimes 2}\right)^{\Sigma_{2}} .
$$

Therefore, Corollary 6.3 follows from Theorem 5.1.

Theorem 6.4. Let $d=2$. Then we have:

$$
H_{k} N \operatorname{Sym}^{2} \Gamma(P .(V)) \cong \begin{cases}\operatorname{Sym}^{2}(V) & \text { for } k=0, \\ \Lambda^{2}(V) \otimes I / I^{2} & \text { for } k=1, \\ D^{2}(V) \otimes \Lambda^{2}\left(I / I^{2}\right) & \text { for } k=2, \\ 0 & \text { for } k \geq 3\end{cases}
$$

Proof. Let $F_{k}$ denote the functor

$$
F_{k}: \mathcal{P}_{R / I} \rightarrow(R \text {-modules }), \quad V \mapsto H_{k} N \operatorname{Sym}^{2} \Gamma(P .(V)) .
$$

Obviously, the functor $F_{k}$ is of degree $\leq 2$. By the Eilenberg-Zilber theorem (see Application 8.5.3 on p. 277 in [W]), we have for all $V, W \in \mathcal{P}_{R / I}$,

$$
\begin{aligned}
\operatorname{cr}_{2}\left(F_{k}\right)(V, W) \cong & H_{k} N(\Gamma(P .(V)) \otimes \Gamma(P .(W))) \\
& \cong H_{k} \operatorname{Tot}(P .(V) \otimes P .(W)) \cong \operatorname{Tor}_{k}^{R}(V, W) .
\end{aligned}
$$

Furthermore, we have $\operatorname{Tor}_{k}^{R}(R / I, R / I) \cong \Lambda^{k}\left(I / I^{2}\right)$ by Example 5.2. Therefore, by Proposition 1.8, Corollary 1.6, Proposition 1.9, and Example 1.7, it suffices to show that the plus map $+_{2}: \operatorname{cr}_{2}\left(F_{0}\right)(R / I, R / I) \rightarrow F_{0}(R / I)$ is bijective, $F_{1}(R / I)$ vanishes, the diagonal map $\Delta_{2}: F_{2}(R / I) \rightarrow \mathrm{cr}_{2}(R / I, R / I)$ is bijective, and $F_{k}(R / I)$ vanishes for $k \geq 3$. For this, we may assume that the ideal $I$ is globally generated by a regular sequence, say $I=(f, g)$. Let $K$. denote the complex $\ldots \rightarrow 0 \rightarrow R \stackrel{f}{\rightarrow} R$ and $L$. the complex $\ldots \rightarrow 0 \rightarrow R \stackrel{g}{\rightarrow} R$. Then,

$$
\operatorname{Tot}(K . \otimes L .) \cong \operatorname{Kos}^{2}(R \oplus R \stackrel{(f, g)}{\longrightarrow} R)
$$

is a resolution of $R / I$. For any bisimplicial $R$-module $M$.., let $\Delta M$.. denote the simplicial $R$-module $\Delta^{\mathrm{op}} \rightarrow(R$-modules $),[n] \mapsto M_{n n}$. The Dold-Kan correspondence (see Proposition 2.1) and the Eilenberg-Zilber theorem yield natural isomorphisms

$$
\begin{aligned}
& F_{k}(R / I) \cong H_{k} N \operatorname{Sym}^{2} \Gamma \operatorname{Tot}(K . \otimes L .) \cong H_{k} N \operatorname{Sym}^{2} \Gamma \operatorname{Tot}(N \Gamma(K .) \otimes N \Gamma(L .)) \\
& \quad \cong H_{k} N \operatorname{Sym}^{2} \Gamma N \Delta(\Gamma(K .) \otimes \Gamma(L .)) \cong H_{k} N \operatorname{Sym}^{2} \Delta(\Gamma(K .) \otimes \Gamma(L .)) \\
& \quad \cong H_{k} N \Delta \operatorname{Sym}^{2}(\Gamma(K .) \otimes \Gamma(L .)) .
\end{aligned}
$$


For any two f. g. projective $R$-modules $P, Q$, the following sequence is exact:

$$
0 \rightarrow \Lambda^{2}(P) \otimes \Lambda^{2}(Q) \rightarrow \operatorname{Sym}^{2}(P \otimes Q) \rightarrow \operatorname{Sym}^{2}(P) \otimes \operatorname{Sym}^{2}(Q) \rightarrow 0
$$

(see p. 242 in $[\mathrm{ABW}]$ ); here, the first map is given by

$$
p_{1} \wedge p_{2} \otimes q_{1} \wedge q_{2} \mapsto\left(p_{1} \otimes q_{1}\right)\left(p_{2} \otimes q_{2}\right)-\left(p_{1} \otimes q_{2}\right)\left(p_{2} \otimes q_{1}\right),
$$

and the second map is given by $\left(p_{1} \otimes q_{1}\right)\left(p_{2} \otimes q_{2}\right) \mapsto p_{1} p_{2} \otimes q_{1} q_{2}$. Thus, we obtain the following short exact sequence of bisimplicial $R$-modules:

$$
\Lambda^{2} \Gamma(K .) \otimes \Lambda^{2} \Gamma(L .) \longleftrightarrow \operatorname{Sym}^{2}(\Gamma(K .) \otimes \Gamma(L .)) \longrightarrow \operatorname{Sym}^{2} \Gamma(K .) \otimes \operatorname{Sym}^{2} \Gamma(L .) ;
$$

applying $N \Delta$ to this sequence then yields a short exact sequence of complexes. By the Eilenberg-Zilber theorem and Proposition 2.4, we have

$$
\begin{array}{rl}
H_{k} & N \Delta\left(\operatorname{Sym}^{2} \Gamma(K .) \otimes \operatorname{Sym}^{2} \Gamma(L .)\right) \\
& \cong H_{k} \operatorname{Tot}\left(N \operatorname{Sym}^{2} \Gamma(K .) \otimes N \operatorname{Sym}^{2} \Gamma(L .)\right) \\
\cong & H_{k} \operatorname{Tot}\left(\operatorname{Kos}^{2}(f) \otimes \operatorname{Kos}^{2}(g)\right) \\
& \cong H_{k} \operatorname{Tot}(K . \otimes L .) \\
& \cong \begin{cases}R / I & \text { for } k=0, \\
0 & \text { for } k \geq 1 .\end{cases}
\end{array}
$$

Similarly, we obtain (cf. Remark 2.7)

$$
H_{k} N \Delta\left(\Lambda^{2} \Gamma(K .) \otimes \Lambda^{2} \Gamma(L .)\right) \cong \begin{cases}R / I & \text { for } k=2 \\ 0 & \text { for } k \neq 2 .\end{cases}
$$

This finally implies

$$
F_{k}(R / I) \cong \begin{cases}R / I & \text { for } k=0,2 \\ 0 & \text { for } k=1 \text { or } k \geq 3 .\end{cases}
$$

In particular, this finishes the proof of Theorem 6.4 in the cases $k=1$ and $k \geq$ 3. Next, we prove that the diagonal map $\Delta_{2}: F_{2}(R / I) \rightarrow \mathrm{cr}_{2}\left(F_{2}\right)(R / I, R / I)$ is bijective. As above, we have an isomorphism

$$
\left.\operatorname{cr}_{2}\left(F_{2}\right)(R / I, R / I) \cong H_{2} N(\Gamma(\operatorname{Tot}(K . \otimes L .)))^{\otimes 2}\right) \cong H_{2} N \Delta\left((\Gamma(K .) \otimes \Gamma(L .))^{\otimes 2}\right)
$$

such that the following diagram commutes:

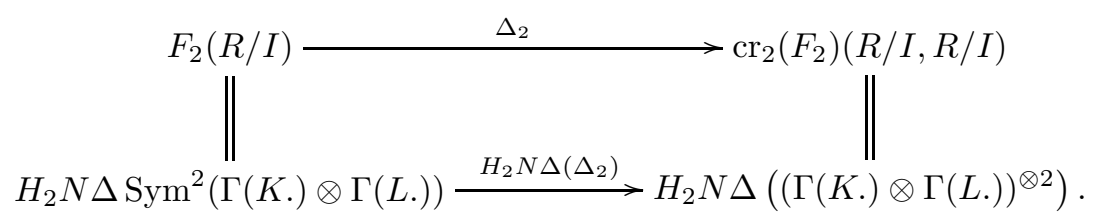

Furthermore, we have the commutative diagram of bisimplicial $R$-modules

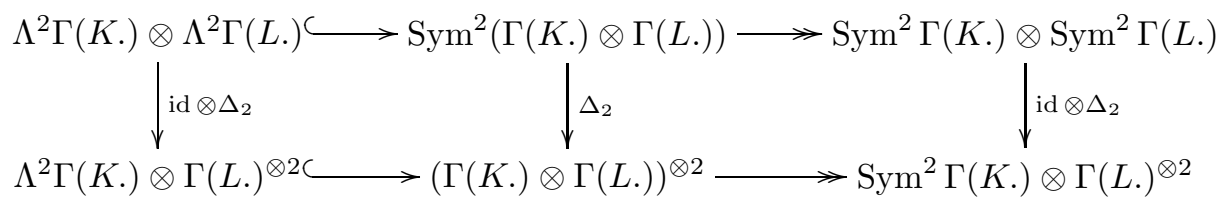

where the upper row is the exact sequence already constructed above and the lower row is induced by the natural exact sequence

$$
0 \rightarrow \Lambda^{2} \Gamma(K .) \rightarrow \Gamma(K .)^{\otimes 2} \rightarrow \operatorname{Sym}^{2} \Gamma(K .) \rightarrow 0 .
$$


Since both $\mathrm{cr}_{2}\left(F_{2}\right)(R / I, R / I)$ and $F_{2}(R / I)$ have rank 1 , it therefore suffices to show that the map

$$
H_{2} N \Delta\left(\mathrm{id} \otimes \Delta_{2}\right): H_{2} N \Delta\left(\Lambda^{2} \Gamma(K .) \otimes \Lambda^{2} \Gamma(L .)\right) \rightarrow H_{2} N \Delta\left(\Lambda^{2} \Gamma(K .) \otimes \Gamma(L .)^{\otimes 2}\right)
$$

is surjective and that $H_{2} N \Delta\left(\operatorname{Sym}^{2} \Gamma(K.) \otimes \Gamma(L .)^{\otimes 2}\right) \cong 0$. As above, we have an isomorphism

$$
\begin{aligned}
& H_{2} N \Delta\left(\Lambda^{2} \Gamma(K .) \otimes \Gamma(L .)^{\otimes 2}\right) \cong H_{2} \operatorname{Tot}\left(\tilde{\operatorname{Kos}^{2}}(K .) \otimes L .{ }^{\otimes 2}\right) \\
& \quad \cong H_{2} \operatorname{Tot}\left(K .[-1] \otimes L .{ }^{\otimes 2}\right) \cong H_{1}\left((R /(f) \stackrel{\bar{g}}{\rightarrow} R /(f))^{\otimes 2}\right) \cong(\bar{g}) /(\bar{g})^{2} \cong R / I .
\end{aligned}
$$

(Note that $\bar{g}$ is a nonzero-divisor in $R /(f)$.) Similarly, we get

$$
H_{2} N \Delta\left(\operatorname{Sym}^{2} \Gamma(K .) \otimes \Gamma(L .)^{\otimes 2}\right) \cong 0 .
$$

The homology class of the element $(1 \otimes 1) \otimes(1 \otimes 1) \in\left(K_{1} \otimes K_{0}\right) \otimes\left(L_{1} \otimes L_{0}\right) \cong$ $\operatorname{Tot}\left(\tilde{\operatorname{Kos}}^{2}(f) \otimes \tilde{\operatorname{Kos}}^{2}(g)\right)_{2}$ obviously generates $H_{2} \operatorname{Tot}\left(\tilde{\operatorname{Kos}^{2}}(f) \otimes \tilde{\operatorname{Kos}}^{2}(g)\right)$. The map id $\otimes \Delta_{2}$ maps this element to the element $(1 \otimes 1) \otimes(1 \otimes 1,-1 \otimes 1)$ of

$$
\begin{aligned}
\left(K_{1}\right. & \left.\otimes K_{0}\right) \otimes\left(L_{1} \otimes L_{0} \oplus L_{0} \otimes L_{1}\right) \\
& \cong \tilde{\operatorname{Kos}^{2}}(K .)_{1} \otimes\left(L .{ }^{\otimes 2}\right)_{1} \subseteq \operatorname{Tot}\left(\tilde{\operatorname{Kos}^{2}}(K .) \otimes L .{ }^{\otimes 2}\right)_{2} .
\end{aligned}
$$

The homology class of $(1 \otimes 1) \otimes(1 \otimes 1,-1 \otimes 1)$ obviously generates $H_{2} \operatorname{Tot}\left(\tilde{\operatorname{Kos}^{2}}(K.) \otimes\right.$ $\left.L .{ }^{\otimes 2}\right)$. This finishes the proof of Theorem 6.4 in the case $k=2$. Similarly, one can show that the plus map $+_{2}: \operatorname{cr}_{2}\left(F_{0}\right)(R / I, R / I) \rightarrow F_{0}(R / I)$ is bijective. Then, the proof of Theorem 6.4 is complete. However, this final case can also be proved directly as follows:

$$
\begin{aligned}
H_{0} N \operatorname{Sym}^{2} \Gamma(P .(V)) & \cong \operatorname{coker}\left(\operatorname{Sym}^{2}\left(P_{1}\right) \oplus P_{1} \otimes P_{0} \stackrel{d_{0}-d_{1}}{\longrightarrow} \operatorname{Sym}^{2}\left(P_{0}\right)\right) \\
& \cong \operatorname{coker}\left(P_{1} \otimes P_{0} \stackrel{\text { can }}{\longrightarrow} \operatorname{Sym}^{2}\left(P_{0}\right)\right) \cong \operatorname{Sym}^{2}(V)
\end{aligned}
$$

(see also Remark 3.3(b)).

Remark 6.5. For any functor $F: \mathcal{P}_{R} \rightarrow \mathcal{P}_{R}$ (with $F(0)=0$ ) which commutes with localization, the support of the homology modules $H_{k} N F \Gamma(P .(V)), k \geq 0$, is contained in $V(I)=\operatorname{Spec}(R / I)$ since the complex $\left.N F \Gamma(P .(V))\right|_{\operatorname{Spec}(R) \backslash V(I)}$ is homotopic to the zero complex (cf. section 2). In contrast to Theorem 3.2, Theorem 5.1 , and Theorem 6.4, these homology modules need not to be projective $R / I$ modules, they even need not to be annihilated by $I$ in general. For instance, if $d=1$, the natural short exact sequence of complexes

$$
0 \rightarrow N D^{2} \Gamma(P .(V)) \rightarrow N\left(\Gamma(P .(V))^{\otimes 2}\right) \rightarrow N \Lambda^{2} \Gamma(P .(V)) \rightarrow 0
$$

together with Remark 3.6 and Example 5.2 yield the long exact sequence

$$
\begin{aligned}
0 \rightarrow & H_{1} N D^{2} \Gamma(P .(V)) \rightarrow V^{\otimes 2} \otimes I / I^{2} \rightarrow D^{2}(V) \otimes I / I^{2} \\
& \rightarrow H_{0} N D^{2} \Gamma(P .(V)) \rightarrow V^{\otimes 2} \rightarrow \Lambda^{2}(V) \rightarrow 0,
\end{aligned}
$$

and one easily sees that $H_{0} N D^{2} \Gamma(P .(R / I)) \cong R / I^{2}$ if $\operatorname{char}(R)=2$. If $d=2$, the natural short exact sequence of complexes

$$
0 \rightarrow N \Lambda^{2} \Gamma(P .(V)) \rightarrow N\left(\Gamma(P .(V))^{\otimes 2}\right) \rightarrow N \operatorname{Sym}^{2} \Gamma(P .(V)) \rightarrow 0
$$


together with Theorem 6.4 and Example 5.2 yield the long exact sequence

$$
\begin{aligned}
0 \rightarrow & H_{2} N \Lambda^{2} \Gamma(P .(V)) \rightarrow V^{\otimes 2} \otimes \Lambda^{2}\left(I / I^{2}\right) \rightarrow D^{2}(V) \otimes \Lambda^{2}\left(I / I^{2}\right) \\
& \rightarrow H_{1} N \Lambda^{2} \Gamma(P .(V)) \rightarrow V^{\otimes 2} \otimes I / I^{2} \rightarrow \Lambda^{2}(V) \otimes I / I^{2} \\
& \rightarrow \Lambda^{2}(V) \rightarrow V^{\otimes 2} \rightarrow \operatorname{Sym}^{2}(V) \rightarrow 0 .
\end{aligned}
$$

One can show that the map $V^{\otimes 2} \otimes \Lambda^{2}\left(I / I^{2}\right) \rightarrow D^{2}(V) \otimes \Lambda^{2}\left(I / I^{2}\right)$ is $+_{2} \otimes$ id. Hence, $H_{k} N \Lambda^{2} \Gamma(P .(V))$ has non-trivial 2-torsion for $k=1,2$. Similarly, one may expect that $H_{k} N \operatorname{Sym}^{2} \Gamma(P .(V))$ can have non-trivial 2-torsion if $d \geq 3$ and $k \geq 2$. This is also suggested by the calculation of the stable derived functors of $\mathrm{Sym}^{2}$ (e.g., see Theorem 4.7 in $[\mathrm{JM}]$ ).

Example 6.6. Let $K$ be a $\lambda$-ring, $C \in K$ of $\lambda$-degree 2, and $x \in K$, arbitrary. An easy, but somewhat tedious calculation shows the following representation of $\sigma_{3}(C, x)$ :

$$
\begin{aligned}
\sigma_{3}(C, x)= & \sigma_{3}(x)-s_{1}^{3}(x) C+\left(\sigma_{2}(x) x \lambda_{2}(C)+\lambda_{3}(x) \sigma_{2}(C)\right) \\
& -s_{1}^{3}(x) C \lambda_{2}(C)+\sigma_{3}(x) \lambda_{2}(C)^{2} .
\end{aligned}
$$

In view of Theorem 5.1 and Theorem 6.4, this suggests the following conjecture: If $d=2$, then we have

$$
H_{k} N \operatorname{Sym}^{3} \Gamma(P .(V)) \cong \begin{cases}\operatorname{Sym}^{3}(V) & \text { for } k=0, \\ L_{1}^{3}(V) \otimes I / I^{2} & \text { for } k=1, \\ \tilde{L}_{1}^{3}(V) \otimes I / I^{2} \otimes \Lambda^{2}\left(I / I^{2}\right) & \text { for } k=3, \\ D^{3}(V) \otimes \Lambda^{2}\left(I / I^{2}\right)^{\otimes 2} & \text { for } k=4, \\ 0 & \text { for } k \geq 5,\end{cases}
$$

and there is a short exact sequence

$$
0 \rightarrow D^{2}(V) \otimes V \otimes \Lambda^{2}\left(I / I^{2}\right) \rightarrow H_{2} N \operatorname{Sym}^{3} \Gamma(P .(V)) \rightarrow \Lambda^{3}(V) \otimes \operatorname{Sym}^{2}\left(I / I^{2}\right) \rightarrow 0 .
$$

\section{REFERENCES}

[ABW] K. Akin, D. A. Buchsbaum, and J. Weyman, Schur functors and Schur complexes, Adv. Math. 44 (1982), 207-278. MR 84c:20021

[AT] M. F. AtiYah and D. O. TAll, Group representations, $\lambda$-rings and the $J$-homomorphism, Topology 8 (1969), 253-297. MR 39:5702

[SGA 6] P. Berthelot, A. Grothendieck, and L. Illusie, "Théorie des Intersections et Théorème de Riemann-Roch", Lecture Notes in Math. 225 (Springer, Berlin, 1971). MR 50:7133

[DP] A. Dold and D. Puppe, Homologie nicht-additiver Funktoren. Anwendungen, Ann. Inst. Fourier Grenoble 11 (1961), 201-312. MR 27:186

[E] S. Eilenberg, Abstract description of some basic functors, J. Indian Math. Soc. 24 (1960), 231-234. MR 23:A2454

[EM] S. Eilenberg and S. Mac Lane, On the groups $H(\pi, n)$, II, Ann. of Math. 60 (1954), 49-139. MR 16:391

[FL] W. Fulton and S. LAng, "Riemann-Roch algebra", Grundlehren Math. Wiss. 277 (Springer, New York, 1985). MR 88h:14011

[G] D. R. Grayson, Adams operations on higher K-theory, K-theory 6 (1992), 97-111. MR 94g:19005

[I1] L. Illusie, "Complexe Cotangent et Déformations I", Lecture Notes in Math. 239 (Springer, Berlin, 1971). MR 58:10886a

[I2] _ "Complexe Cotangent et Déformations II", Lecture Notes in Math. 283 (Springer, Berlin, 1972). MR 58:10886b

[JM] B. Johnson and R. McCarthy, Linearization, Dold-Puppe stabilization, and Mac Lane's Q-construction, Trans. Amer. Math. Soc. 350 (1998), 1555-1593. MR 98i:18007 
[J] J. P. Jounanolou, Riemann-Roch sans dénominateurs, Invent. Math. 11 (1970), 15-26. MR 48:11115

[K 1] B. Köck, The Grothendieck-Riemann-Roch theorem for group scheme actions, Ann. Sci. École Norm. Sup. 31 (1998), 415-458. MR 99f:14010

[K 2] Riemann-Roch for tensor powers, Math. Z. 233 (2000), 755-801. CMP 2000:13

[M] Y. I. Manin, Lectures on the $K$-functor in algebraic geometry, Russian Math. Surveys 24, No. 5 (1969), 1-89. MR 42:265

[S] C. SoulÉ, Opérations en K-théorie algébrique, Canad. J. Math. 37, No. 3 (1985), 488550. MR 87b:18013

[TT] R. W. Thomason and T. Trobaugh, Higher algebraic $K$-theory of schemes and of derived categories, in: "The Grothendieck Festschrift, Vol. III", Progr. Math. 88 (Birkhäuser, Boston, 1990), 247-435. MR 92f:19001

[Wa] C. E. Watts, Intrinsic characterizations of some additive functors, Proc. Amer. Math. Soc. 11 (1960), 5-8. MR 22:9528

[W] C. A. Weibel, "An introduction to homological algebra", Cambridge Stud. Adv. Math. 38 (Cambridge University Press, Cambridge, 1994). MR 95f:18001

Mathematisches Institut II, Universität Karlsruhe, 76128 Karlsruhe, Germany

Current address: Faculty of Mathematical Studies, University of Southampton, Southampton SO17 1BJ, United Kingdom

E-mail address: Bernhard.Koeck@math.uni-karlsruhe.de 